\title{
Ultrasound-based density determination via buffer rod techniques: a review
}

\author{
S. Hoche, M. A. Hussein, and T. Becker \\ Chair of Brewing and Beverage, Bio-PAT (Bio-Process Analysis Technology), Freising, Germany \\ Correspondence to: S. Hoche (s.hoche@wzw.tum.de)
}

Received: 18 March 2013 - Revised: 18 June 2013 - Accepted: 8 July 2013 - Published: 31 July 2013

\begin{abstract}
The review presents the fundamental ideas, assumptions and methods of non-invasive density measurements via ultrasound at solid-liquid interface. Since the first investigations in the 1970s there has been steady progress with regard to both the technological and methodical aspects. In particular, the technology in electronics has reached such a high level that industrial applications come within reach. In contrast, the accuracies have increased slowly from $1-2 \%$ to $0.15 \%$ for constant temperatures and to $0.4 \%$ for dynamic temperature changes. The actual work reviews all methodical aspects, and highlights the lack of clarity in major parts of the measurement principle: simplifications in the physical basics, signal generation and signal processing. With respect to process application the accuracy of the temperature measurement and the presence of temperature gradients have been identified as a major source of uncertainty. In terms of analytics the main source of uncertainty is the reflection coefficient, and as a consequence of this, the amplitude accuracy in time or frequency domain.
\end{abstract}

\section{Introduction}

The medium density is a key parameter for most known processes in chemical, petrochemical, pharmaceutical, food and beverage, biotechnology, water and waste-water industries. The potential to determine online the quantity and quality of the process medium by means of density enables new options of process control and management. There are methods based on direct physical relations or based on the determination of parameters that can be correlated to the density for a specific chemical reaction or a characteristic process course. But most established methods, like coriolis mass flow or vibrating U-tube, have system-inherent limitations that often result in application restrictions in sensor implementation (limits in pipe diameter, limited to bypass application, limited to a certain flow range). Based on the specifications of the process, additional limitations might be sensitivity to bubbles, particles or fouling. In the case of food processing, hygienic design is a dominant constraint. The actual paper reviews ultrasound-based techniques as alternative methods which may be used where standard methods are not applicable.
The easiest way to determine the real-time density is to monitor the ultrasound velocity. According to the NewtonLaplace equation

$\kappa_{\mathrm{S}}=\frac{1}{\rho_{1} c_{1}^{2}}$,

the density $\rho_{1}$ of a liquid medium can be determined knowing the isentropic (adiabatic) compressibility $\kappa_{\mathrm{S}}$ and the sound velocity $c_{1}$. Unfortunately, the adiabatic compressibility is usually determined from sound velocity and density measurements at atmospheric pressure (Kaatze et al., 2008). In 1967 Davis and Gordon (Davis and Gordon, 1967) developed an exact method to measure the adiabatic compressibility by determining volume and sound velocity changes under varying pressure and temperature. Davis and Gordon's research work was followed by extensive investigations to determine thermophysical properties of different materials (Bolotnikov et al., 2005; Daridon et al., 1998a, b; Esperança et al., 2006; Kell, 1975; Żak et al., 2000). Since all three parameters - density, sound velocity and compressibility - are highly temperature dependent, and since the compressibility measurement is limited to laborious methods, the application of sonic velocimetry at constant frequencies is limited to 
density determination of binary systems (Asher, 1987; Van Sint Jan et al., 2008). The velocimetric approach is based on temperature and, in some cases, pressure-dependent calibration measurements of sufficiently pure and well-defined liquids (Rychagov et al., 2002) and results in applications such as electrolyte measurements in accumulators or density determination of pure liquids (Swoboda et al., 1983; Vray et al., 1992; Wang et al., 2011; Kuo, 1971; Marks, 1976; Wang and Nur, 1991). The accuracy of such methods generally depends on the type of liquid and its purity (Rychagov et al., 2002; Matson et al., 2002; Wang and Nur, 1991).

Further methods to determine the density via ultrasound are waveguide and interferometric approaches. The waveguide approach generally uses propagation time variations of torsional ultrasonic waves in a transmission line immersed in the sample liquid. Besides torsional waves, the use of flexural or Rayleigh waves is also possible. Even though waveguide sensors have been used by several research groups over the last decades (Kim and Bau, 1989), it is reported (Lynnworth, 1994) that the method suffers from viscosity effects and has to be specifically designed to fulfil certain wavelength aspects.

The interferometric approaches use the effects of overlapping waves. While Pope et al. $(1992,1994)$ used peak FFT values of the resonance response spectrum over a certain frequency range, Sinha and Kaduchak (Sinha and Kaduchak, 2001; Kaduchak and Sinha, 2001; Sinha, 1998) used sweptfrequency acoustic interferometry (SFAI) based on characteristics of standing-wave patterns. Pope's method relies on calibration measurements, and therefore is limited in the same way as the velocimetric methods. The method presented by Sinha and Kaduchak was not developed for highly accurate acoustic measurements. They reported a relative uncertainty of $0.5 \%$ for sound speed and $5 \%$ for the density measurement.

In conclusion to the text above, one can allege that the enormous calibration effort of most ultrasound-based methods may be the reason that, in the past decades, several research groups have focused on reflection-coefficient-based density determination methods via buffer rod systems. The plane wave propagation across one or more interface is the basis of buffer rod techniques. The history of single pulses is described with respect to the excitation amplitude considering reflection, transmission and attenuation terms. Calculating the ratios of feasible pulses results in amplitude-based representation of the reflection coefficient. Further parameters like attenuation and density can be calculated based on the knowledge of the buffer material's properties.

Sachse (1974) and Hale (1988) first reported on this method and presented validation results. Sachse analysed the amplitudes of pulses, scattered by a fluid-filled inclusion in an aluminium block to determine the reflection coefficient (RC), $r$ of the pulse incident on the inclusion. Finally, the measured RC and the known impedance of the matrix material were used to calculate the density of the inclusion fluid.
In contrast, Hale used a transmitter-receiver configuration. From the amplitude changes of received signals, he determined the sample density with a bias of less than $2 \%$.

McClements and Fairly $(1991,1992)$ first paid attention to attenuation and temperature effects for their validation trials. The developed ultrasonic pulse echo reflectometer consists of a perspex buffer rod and an aluminium reflector plate. The reflectometer has been immersed in a water bath to stabilize the temperature to $\pm 0.1^{\circ} \mathrm{C}$. According to Eq. (2) the $\mathrm{RC}, r_{\text {buffer-sample }}$ of the interface perspex buffer-sample-fluid was calculated by the use of reference signals, for which the reference medium was air. Assuming total reflection $\left(Z_{\text {air }} \ll\right.$ $Z_{\text {perspex }} ; r \approx 1$ ) and constant incident pulse amplitudes $A_{\mathrm{i}}$ the ratio of the first echo's amplitudes leads to an attenuation independent term:

$r_{\text {buffer-sample }}=A_{1 \text { sample }} / A_{1 \text { air }}$,

where $A_{1 \text { sample }}$ is the pulse amplitude of the first pulse that is reflected from buffer-sample-fluid interface and $A_{1 \text { air }}$ is the pulse amplitude of the first pulse that is reflected from buffer-air interface of the reference measurement. Knowing the RC $r_{\text {buffer-sample, }}$, the specific acoustic impedance of the actual sample can be determined. McClements and Fairly achieved remarkable accuracy of $\pm 0.01 \times 10^{6} \mathrm{~kg} \mathrm{~m}^{-2} \mathrm{~s}^{-1}$ for the impedance determination. A precision of approximately $\pm 0.5 \mathrm{~m} \mathrm{~s}^{-1}$ was reported for the speed-of-sound measurements. Using both to calculate densities for a series of sodium chloride solutions, an accuracy of $\pm 6 \mathrm{~kg} \mathrm{~m}^{-3}(0.5 \%)$ could be achieved.

In general, all subsequent investigations are based upon the same basic relations, only varying in sensor design, methodology adaptions and signal analysis. The review focuses on ultrasound-based density determination via buffer rod techniques (BRT). In Sect. 2 the physical fundamentals and basic assumptions will be discussed as well as the four basic methods that have been identified. In Sect. 3 relevant design considerations will be presented. Finally, in Sect. 4, all major analytical aspects will be discussed with respect to density accuracy, uncertainties and real process application.

\section{Physical fundamentals and method classification}

The basis of all BRTs is the determination of the RC, which in general is based upon the physical description of plane wave propagation across an interface (see Fig. 1). Every medium is characterized by certain sound velocity $c$, density $\rho$ and sound attenuation $\alpha$. Any loss of energy that appears while sound wave propagates through homogeneous medium is summarized in the attenuation term. As soon as the wave arrives at an interface, the wave will be partly transmitted and partly reflected.

The relation of transmission and reflection is governed by the specific acoustic impedance $Z$ of the medium defined as

$Z=\frac{\omega}{k} \rho=\frac{\omega}{\omega / c-j \alpha} \rho=\frac{c}{1-j \alpha^{c} / \omega} \rho$, 


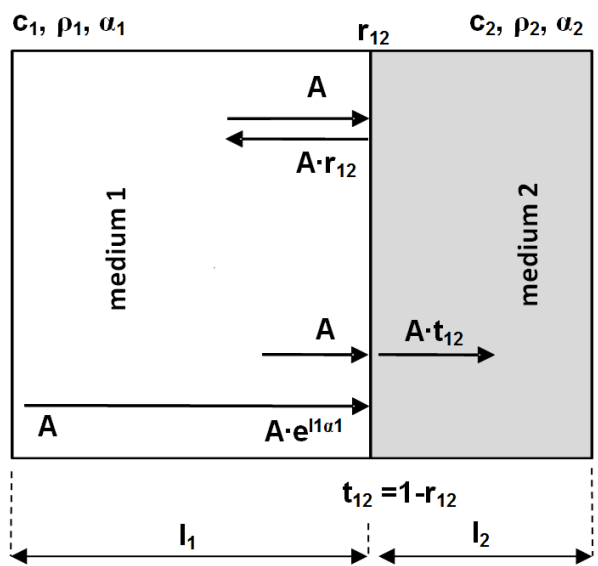

Figure 1. Schema showing the basic principles of sound propagation across an interface at normal incidence.

where $k$ is the complex wave number and $\omega$ the angular frequency $(=2 \pi f)$. For materials of sufficiently small attenuation $(\alpha \ll \omega / c$ or $\alpha c / \omega \ll 1)$, Eq. (3) simplifies to

$Z=\rho \cdot c$.

The amount of a wave reflected at a plane interface is often characterized by the RC which is the ratio of the reflected (subscript $r$ ) to the incident (subscript $\mathrm{i}$ ) wave. The RC can be expressed in terms of amplitudes A or intensities I. The intensity is proportional to the square of amplitude, which leads to the following expressions for a wave that passes from medium 1 (subscript 1) to medium 2 (subscript 2):

$r_{\mathrm{A}}=\frac{A_{\mathrm{r}}}{A_{\mathrm{i}}}=\frac{Z_{2}-Z_{1}}{Z_{2}+Z_{1}}$

$r_{\mathrm{I}}=\frac{I_{\mathrm{r}}}{I_{\mathrm{i}}}=\left(\frac{Z_{2}-Z_{1}}{Z_{2}+Z_{1}}\right)^{2}$

In the same way the transmission coefficient $t$ is given as the ratio of transmitted wave (subscript $t$ ) to incident wave:

$t_{\mathrm{A}}=1-r_{\mathrm{A}}=\frac{A_{\mathrm{t}}}{A_{\mathrm{i}}}=\frac{2 Z_{1}}{Z_{2}+Z_{1}}$.

If one thinks in terms of buffer rod techniques (BRTs), medium 1 might be the buffer rod and medium 2 the sample liquid. Measuring at constant temperatures, the material properties $(c$ and $\rho$ ) of the buffer remains constant, and any change in the RC is clearly related to a change of the specific acoustic impedance of the sample liquid. This means according to Eqs. (4)-(6), the density of the sample liquid $\rho_{2}$ can be determined via the reflection coefficient if the temperaturedependent properties of the buffer rod $\left(\rho_{1}, c_{1}\right)$ and the sound velocity of the sample liquid $\left(c_{2}\right)$ are known:

$\rho_{2}=\frac{\rho_{1} c_{1}}{c_{2}} \frac{\left(1+r_{\mathrm{A}}\right)}{\left(1-r_{\mathrm{A}}\right)}=\frac{\rho_{1} c_{1}}{c_{2}} \frac{\left(1+r_{\mathrm{I}}^{2}\right)}{\left(1-r_{\mathrm{I}}^{2}\right)}$.
The wave propagation in its basic form is a mechanical oscillation and depends on the physical properties of the material (Saggin and Coupland, 2001; McClements, 1997; Povey and McClements, 1988):

$\left(\frac{k}{\omega}\right)^{2}=\frac{\rho}{\text { modulus of elasticity }}$.

In the case of pressure waves, the appropriate modulus of elasticity is the longitudinal modulus $\mathrm{M}$, which is equal to the sum of bulk modulus $\mathrm{K}$ and $4 / 3$ shear modulus G. For Newtonian fluids the shear modulus can be neglected and the modulus of elasticity is assumed to be equal to the bulk modulus $\mathrm{K}\left(=\kappa^{-1}\right.$; see Eq. 1$)$. If one considers that the wave number is complex and the attenuation in liquids is not negligible, the acoustic impedance becomes complex, expressed as the complex sum of the resistive (real) part, $R_{\mathrm{a}}$, and the reactive (imaginary) part, $X_{\mathrm{a}}$ :

$Z_{\mathrm{a}}=\frac{P}{\xi}=R_{\mathrm{a}}+j X_{\mathrm{a}}$,

where $P$ is the acoustic pressure and $\xi$ the particle displacement. Applying a BRT, the attenuation in the buffer is generally low and the simplification of Eq. (5) is valid. This may change in the case of a fluid as second phase. For high attenuation, a complex form of the RC is introduced which includes a loss angle, $\theta$ (O'Neil, 1949; Mason et al., 1949; Moore and McSkimin, 1970):

$r e^{-j \theta}=\frac{Z_{2}-Z_{1}}{Z_{2}+Z_{1}}$

leading to a complex acoustic impedance for the sample fluid:

$Z_{2}=R_{2}+j X_{2}=Z_{1} \frac{1-r^{2}-j 2 r \sin \theta}{1+r^{2}-2 r \cos \theta}$.

The resistive (real) part then becomes

$R_{2}=Z_{1} \frac{\left(1-r^{2}\right)}{1+r^{2}-2 r \cos \theta}$,

and can be approximated as

$R_{2} \approx Z_{1} \frac{1+r}{1-r}\left[1-\frac{r \theta^{2}}{(1-r)^{2}}\right]=Z_{1} \frac{1+r}{1-r}+O\left(\theta^{2}\right)$.

Typically the acoustic impedance of liquids is less than $0.1(1+j)$ of the buffer impedance, and therefore the loss angle was found not to exceed $5^{\circ}$ (Mason et al., 1949). The loss angle dependent remainder can be neglected and the approximation can be used to specify the resistive component of the liquid's acoustic impedance for most buffer-liquid interfaces.

The buffer rod techniques published so far differ mainly in the way that the RC is determined, but not in the calculation of the density. Consequently, the accuracy of all BRT-density measurements basically depends on both the accuracy of the $\mathrm{RC}$ and the sound velocity measurement. Based upon the applied RC determination method the BRTs can be classified into multiple reflection methods (MRM), reference reflection methods (RRM), transmission methods (TM) and angular reflection methods (ARM). 


\subsection{Multiple reflection method (MRM)}

The MRM (also known as the ABC method) was first devised by Papadakis (1968). He determined the ultrasonic attenuation in a sample and the RC at the buffer-sample interface over a frequency range of 27-45 MHz. In $1972 \mathrm{~Pa}-$ padakis et al. (1973), together with Fowler and Lynnworth, presented further results in the range $0-15 \mathrm{MHz}$ and introduced a diffraction correction. Based upon the work of Mason and Moore and McSkimin, Sachse (1974) applied the same method to determine the density in a range up to $10 \mathrm{MHz}$. Adamowski et al. (1998, 1995), Higuti and Adamowski (2002a) and Bjørndal et al. (2008) used identical principles, but enhanced some methodical aspects to overcome several error influences.

The core idea of the MRM is the use of pulse ratios. If the correct pulses are related to each other, the unwanted attenuation, reflection and transmission terms can be neglected, leaving a term that is only dependent on the $\mathrm{RC}$ of interest. Principally the remaining term is even independent of the initially generated pulse amplitude. In general, a probe design as shown in Fig. 2 is used for the MRM, in which medium 1 resembles the buffer (subscript 1); medium 2, the sample liquid (subscript 2); and medium 3, the reflector (subscript 3) - all of them characterized by a certain $\kappa, \rho$ and $\alpha$. The reflection or transmission coefficients of the different interfaces are indicated in terms of propagation direction and involved mediums; for example,

$\mathrm{RC}$ for propagation from medium 1 to medium 2 :

$r_{12}=\frac{Z_{2}-Z_{1}}{Z_{2}+Z_{1}}$

transmission coefficient for propagation from medium 2

to medium 1: $\mathrm{t}_{21}=\frac{2 \mathrm{Z}_{2}}{\mathrm{Z}_{1}+\mathrm{Z}_{2}}$.

Using the principles of plane wave propagation at normal incidence, one obtains the following for $A_{\mathrm{r} 1}, A_{\mathrm{e} 11}$ and $A_{\mathrm{e} 21}$ :

$A_{\mathrm{r} 1}=A_{\mathrm{T}} \cdot r_{12} \cdot \exp \left(2 l_{1} \alpha_{1}\right)$

$A_{\mathrm{e} 11}=A_{\mathrm{T}} \cdot t_{12} r_{23} t_{21} \cdot \exp \left(2 l_{1} \alpha_{1}\right) \cdot \exp \left(2 l_{2} \alpha_{2}\right)$,

$A_{\mathrm{e} 21}=A_{\mathrm{T}} \cdot t_{12} r_{23}^{2} r_{21} t_{21} \cdot \exp \left(2 l_{1} \alpha_{1}\right) \cdot \exp \left(4 l_{2} \alpha_{2}\right)$

The subscript $r$ defines the captured pulse as buffer reflection (BR) and the subscript e as an echo pulse. Furthermore in $A_{\mathrm{r} k}$ and $A_{\mathrm{e} j k}$, subscript $k$ defines the pulse order (1st BR, $A_{\mathrm{r} 1}$; 2nd BR, $A_{\mathrm{r} 2}$; etc.) and subscript $j$ the echo order (e.g. pulses of 1 st echo, $A_{\mathrm{e} 1 k}$; pulses of 2 nd echo, $\left.A_{\mathrm{e} 2 k}\right)$. For the ratios $A_{\mathrm{r} 1} / A_{\mathrm{e} 11}$ and $A_{\mathrm{e} 11} / A_{\mathrm{e} 21}$ one obtains

$\frac{A_{\mathrm{r} 1}}{A_{\mathrm{e} 11}}=\frac{r_{12}}{t_{12} r_{23} t_{21} \cdot \exp \left(2 l_{2} \alpha_{2}\right)} ; \frac{A_{\mathrm{e} 11}}{A_{\mathrm{e} 21}}=\frac{1}{r_{23} r_{21} \cdot \exp \left(2 l_{2} \alpha_{2}\right)}$.

The terms of attenuation in medium 1 and the initial transmitted amplitude $A_{\mathrm{T}}$ are cancelled out. Additionally, it becomes clear that disregarding the first interface at the coupled

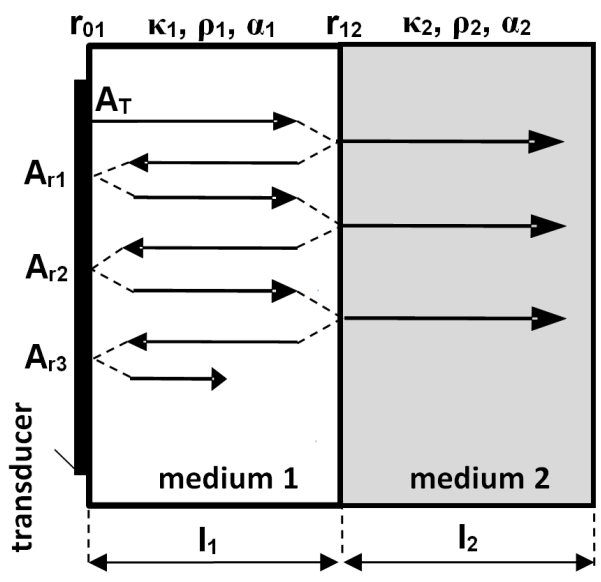

Figure 2. Schematic showing the basic principles and relevant pulses for the MRM: buffer, medium 1; sample, medium 2; reflector, medium 3.

sound source is a valid simplification. Every additional term of the interface $0-1$ (e.g.: $\left.A_{\mathrm{T}}=A_{0} t_{01} t_{10}\right)$ ) would be added to each of the pulses (Eqs. 15, 16 and 17) and therefore also disappear in the ratios of (18).

Dividing now one ratio by the other, one reaches an attenuation-independent equation, and the amplitudes $A_{1}, A_{2}$ and $A_{3}$ can be used to calculate the RC of interface $1-2, r_{12}$ :

$r_{12}=\sqrt{\frac{x}{x-1}} x=\frac{A_{\mathrm{r} 1} A_{\mathrm{e} 21}}{A_{\mathrm{e} 11}^{2}}$.

The resulting equation is now independent of the attenuation in medium 2. Papadakis (1968) first investigated a glass buffer rod on a fused-silica sample. Later, in Papadakis et al. (1973), a water buffer combined with a nickel sample was investigated; a RC of $r_{12}=0.9435 \pm 0.0045$ was calculated, which was in good agreement with the theoretical value of 0.945 . Furthermore, he introduced the so-called A'AB method, which is more or less the first mention of the RRM, and may be used if attenuation in medium 2 is too high and amplitude A3 is very low. Further details about the RRM will follow in the next section.

Instead of the normal buffer-reflector configuration, Adamowski et al. (1995, 1998) used a double-element transducer (DET) including the buffer, a sample liquid (medium 2) and a high-acoustic-impedance reflector (medium 3: stainless steel). The DET has a piezoceramic emitter and a large-aperture receiver (PVDF membrane) separated by a solid buffer rod (medium 1: PMMA) of length $l_{0}$. Another buffer rod (medium 1: PMMA) of length $l_{1}$ is placed between receiver and sample medium. The great advantage of Adamowski's approach is the employment of the largeaperture receiver in the DET. The large aperture minimizes the uncertainties if diffraction effects and the transmitted pulse $A_{\mathrm{T}}$ can be gathered for every single excitation. That enables calibrations due to varying excitation amplitudes as they may occur during long-term operations. Nevertheless 
applying the MRM, the use of $A_{\mathrm{T}}$ is not necessary. In Adamowski et al. (1995) a comparison of MRM and RRM is presented, and for MRM a bias of $10 \mathrm{~kg} \mathrm{~m}^{-3}$ is reported. The main limitation of Adamowski's DET is the PVDF's limited temperature range of application. At temperatures above 60$70^{\circ} \mathrm{C}$ the piezoelectric PVDF slowly loses its imposed polarized structure. A successful application of high-temperature piezoelectric materials (PEM) in a DET has not been reported so far.

Bjørndal et al. (2008) used the MRM to verify a newly developed TM, which will be discussed later. They investigated liquids with a wide range of shear viscosities at a temperature of $27.44 \pm 0.04{ }^{\circ} \mathrm{C}$. It was reported that the systematic deviation from reference values of a calibrated pycnometer was smaller for the MRM than for the TM, and reached an error of $\pm 0.15 \%$.

A special version of the MRM is the approach of Deventer and Delsing (1997). Although this method does not follow the typical ABC approach of Papadakis, it is classified as MRM since some specific reflections are used to calculate the RC without additional calibration measurements. Delsing and Deventer used a double buffer of two different materials. Keeping the terminology of Fig. 2, medium 2 is now the second buffer and medium 3 is the sample liquid. Eliminating $A_{\mathrm{T}}$ in Eq. (17) with the use of Eq. (16) one achieves for $r_{23}$

$r_{23}=\frac{A_{\mathrm{e} 11} \cdot r_{12}}{A_{\mathrm{r} 1} \cdot t_{12} t_{21} \cdot \exp \left(2 l_{2} \alpha_{2}\right)}$,

and for $\rho_{3}$

$\rho_{3}=\frac{Z_{2}}{c_{3}} \cdot \frac{4 A_{\mathrm{r} 1} Z_{1} Z_{2} \exp \left(2 l_{2} \alpha_{2}\right)-A_{\mathrm{e} 11}\left(Z_{1}^{2}-Z_{2}^{2}\right)}{4 A_{\mathrm{r} 1} Z_{1} Z_{2} \exp \left(2 l_{2} \alpha_{2}\right)+A_{\mathrm{e} 11}\left(Z_{1}^{2}-Z_{2}^{2}\right)}$.

Since the properties of medium 1 and 2 are known, the unknown parameters that have to be measured are $c_{3}, A_{\mathrm{e} 11}$ and $A_{\mathrm{r} 1}$. So basically no echo pulse from a reflector is necessary to calculate the RC, which is a great advantage in the case of highly absorptive liquids. The disadvantage is that not only is the exact knowledge of temperature-dependent density and sound velocity of one medium required, but that of two mediums. Additionally, the attenuation in medium 2 has to be known to calculate the RC. And the sound velocity of the sample liquid is still necessary to calculate the density. Therefore transmission or pulse-echo measurements through the liquid are still a requirement to determine the density.

Deventer and Delsing (1997) used 32-times-averaged digitized signals in order to determine the densities of water at 2,20 and $40{ }^{\circ} \mathrm{C}$. The measured densities have been compared with tabulated data, and a mean bias of $1 \mathrm{~kg} \mathrm{~m}^{-3}$ was reported. In fact, the presented graph shows standard deviations from $\pm 5 \mathrm{~kg} \mathrm{~m}^{-3}$ at $40^{\circ} \mathrm{C}$ up to $\pm 10 \mathrm{~kg} \mathrm{~m}^{-3}$ at $2{ }^{\circ} \mathrm{C}$, and it was not mentioned as to how many densities have been averaged to reach the reported results. In Deventer and Delsing (2001a) the densities of glycerin, water and alcohol were determined in a temperature range from 0 to $40^{\circ} \mathrm{C}$. A mean of 100 measurements and tabulated reference data was used for the validation. Even though a clear separation between the results of the different sample liquids is possible, the results still show varying bias and standard deviation for varying temperatures. It was stated that sound velocity inaccuracies generated an error of approximately $1 \%$ and that a density error of $0.4 \%$ should be reachable.

\subsection{Reference reflection method (RRM)}

A first version of the RRM was presented by Papadakis et al. (1973). As with all RRM the core idea is the use of plane wave propagation principles at normal incidence in combination with a reference medium. For the so-called A'AB method, Papadakis uses the 1 st buffer reflection of a reference medium A' and the same 1st buffer reflection of the sample medium A to calculate the RC. The pulse amplitude B is only used to calculate the attenuation. A similar approach was used later by Adamowski et al. (1998), McClements and Fairly (1991), Saggin and Coupland (2001) and Kulmyrzaev et al. (2000).

Similar to the MRM approach of Deventer and Delsing (1997), the RC determination via RRM does not rely on the presence of a reflector. Of course, calculating the final density via Eq. (8) still requires the sound velocity of the sample medium, and therefore needs either transmission or pulseecho measurements through the liquid, but the schematic representation of the basic principles to determine the RC can be simplified to medium 1 and 2 (see Fig. 3). For moderate attenuation and thickness of medium 1 , one can obtain the amplitudes of the multiple buffer reflections $A_{\mathrm{r} k}$ as follows:

$A_{\mathrm{r} 1}=A_{\mathrm{T}} \cdot r_{12} \cdot \exp \left(2 l_{1} \alpha_{1}\right) ; A_{\mathrm{r} 2}=A_{\mathrm{T}} \cdot r_{10} \cdot r_{12}^{2} \cdot \exp \left(4 l_{1} \alpha_{1}\right) ;$

$A_{\mathrm{r} k}=A_{\mathrm{T}} \cdot r_{10}^{k-1} \cdot r_{12}^{k} \cdot \exp \left(2 k l_{1} \alpha_{1}\right)$.

The RRM based on one pulse, as applied in McClements and Fairly (1991), Papadakis et al. (1973), Püttmer and Hauptmann (1998), Püttmer et al. (1998, 2000) and Saggin and Coupland (2001), uses the ratio of any detectable buffer reflection of a sample medium and the corresponding buffer reflection of a reference medium, e.g. $A_{\mathrm{r} 1}$ (sample) and $A_{\mathrm{r} 1}($ reference):

$\frac{A_{\mathrm{r} 1}(\text { sample })}{A_{\mathrm{r} 1}(\text { reference })}=\frac{A_{\mathrm{T}} \cdot r_{12}(\text { sample }) \cdot \exp \left(2 l_{1} \alpha_{1}\right)}{A_{\mathrm{T}} \cdot r_{12}(\text { reference }) \cdot \exp \left(2 l_{1} \alpha_{1}\right)}$.

Assuming a constant excitation pulse $A_{\mathrm{T}}$ and a similar attenuation $\alpha_{1}$ for sample and reference signal one obtains

$r_{12}($ sample $)=r_{12}($ reference $) \frac{A_{\mathrm{r} 1}(\text { sample })}{A_{\mathrm{r} 1}(\text { reference })}$.

The RRM based on two pulses as applied in Adamowski et al. (1998) uses the ratio of any detectable buffer reflection and its following reflection, e.g. $A_{\mathrm{T}}$ and $A_{\mathrm{r} 1}$ or $A_{\mathrm{r} 1}$ and $A_{\mathrm{r} 1}$ :

$\frac{A_{\mathrm{r} 1}(\text { sample }) / A_{\mathrm{r} 2}(\text { sample })}{A_{\mathrm{r} 1}(\text { reference }) / A_{\mathrm{r} 2}(\text { reference })}=\frac{r_{12}(\text { reference })}{r_{12}(\text { sample })}$.

Since successive ratio buffer pulses are used, the excitation pulse $A_{\mathrm{T}}$ does not have to be assumed constant anymore. But 

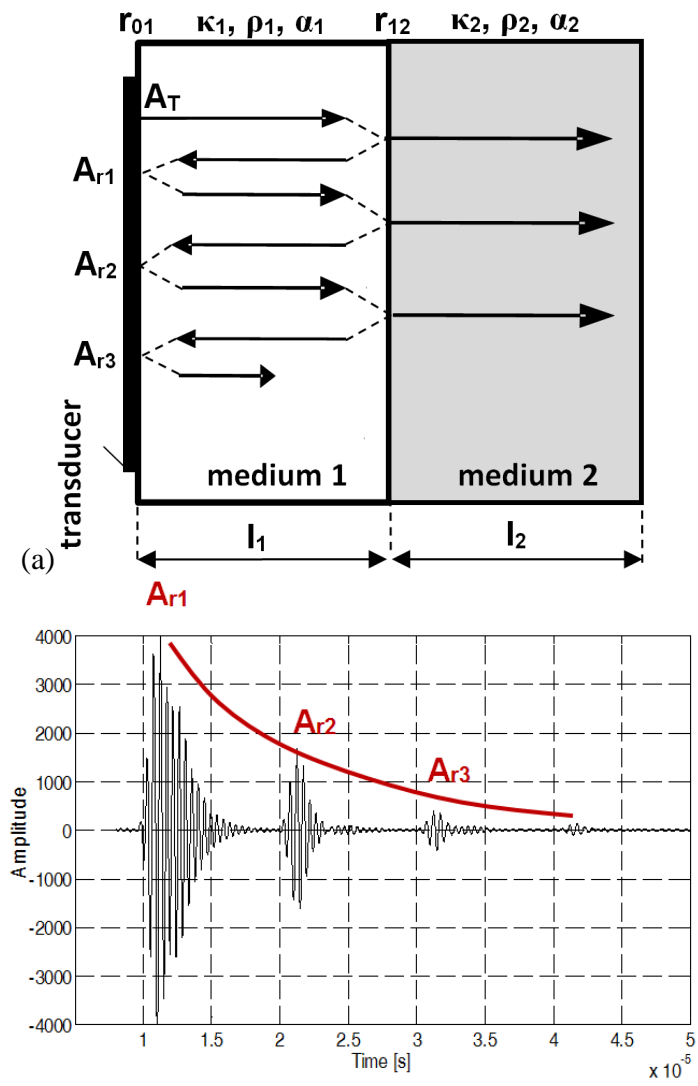

(b)

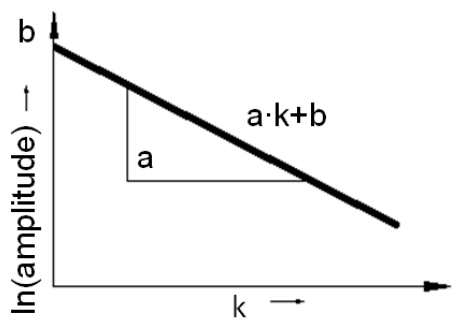

Figure 3. Basic principles and relevant pulses of the RRM: (a) schematic of multiple buffer reflections, (b) multiple buffer reflection pulses in the time domain and logarithmic decay of pulse amplitudes.

still a similar attenuation $\alpha_{1}$ and a similar $\mathrm{RC} r_{10}$ have to be assumed if sample and reference measurement are compared:

$r_{12}($ sample $)=r_{12}($ reference $) \frac{A_{\mathrm{r} 1}(\text { reference }) / A_{\mathrm{r} 2}(\text { reference })}{A_{\mathrm{r} 1}(\text { sample }) / A_{\mathrm{r} 2}(\text { sample })}$.

Since successive ratio buffer pulses are used, the excitation pulse $A_{\mathrm{T}}$ does not have to be assumed constant anymore. But still a similar attenuation $\alpha_{1}$ and a similar RC $r_{10}$ have to be assumed if sample and reference measurement are compared:

$r_{12}($ sample $)=r_{12}($ reference $) \frac{A_{\mathrm{r} 1}(\text { reference }) / A_{\mathrm{r} 2}(\text { reference })}{A_{\mathrm{r} 1}(\text { sample }) / A_{\mathrm{r} 2}(\text { sample })}$

And finally, as applied by Bamberger and Greenwood (2004a, b), the ratio of decays of multiple buffer reflections can be used to obtain the RC via RRM. Describing the amplitude decay logarithmically:

$$
\begin{aligned}
\ln A_{\mathrm{r} k} & =\left[\ln \left(r_{10}\right)+\ln \left(r_{12}\right) \cdot 2 l_{1} \alpha_{1}\right] \cdot k+\left[\ln A_{\mathrm{T}}-\ln \left(r_{10}\right)\right] \\
& =a \cdot k+b,
\end{aligned}
$$

and calculating the ratio $\exp [a$ (sample $)] / \exp [a$ (reference $)]$, one obtains the RC under the assumption of similar attenuation $\alpha_{1}$ and a similar RC $r_{10}$ for reference and sample signals:

$r_{12}($ sample $)=r_{12}($ reference $) \cdot e^{\left[a_{\text {sample }}-a_{\text {reference }}\right]}$.

McClements and Fairly $(1991,1992)$ applied the onepulse RRM with air as the reference medium. They used a $2.1 \mathrm{MHz}$ transducer of $10 \mathrm{~mm}$ diameter driven by a tone burst of 5-10 cycles. Distilled water, castor oil, olive oil, $n$-hexadecane and silicone fluid have been investigated at a constant temperature of $20.2^{\circ} \mathrm{C}$. For a vibrating U-tube as the reference measurement (DMA 40, Anton Paar) an error of $0.5 \%$ is reported, which corresponds to a bias of $\pm 8 \mathrm{~kg} \mathrm{~m}^{-3}$.

Kushibiki et al. (1995) applied a one-pulse RRM to investigate the acoustic properties of biological tissue and liquid specimen. Instead of air, water was used as the reference medium. Kushibiki et al. used a transmission line to measure velocity dispersion and attenuation. Basically the methodological assembly is comparable to Bjørndal's MRM approach. It was not mentioned why an RRM instead of an MRM was applied. Several broadband transducers (1.5 mm diameter) in combination with different gap distances have been used to cover the frequency range from 70 to $500 \mathrm{MHz}$. Different oils have been investigated and a maximum bias of $8 \mathrm{~kg} \mathrm{~m}^{-3}$ is reported. The temperature was reasonably constant around $23^{\circ} \mathrm{C}$, and the density validation values have been gathered via pycnometer. The investigations of Kushibiki et al. particularly show the feasibility of the method to investigate properties of very thin specimen.

Adamowski et al. (1998) applied the two-pulse RRM. Due to the special DET design it was possible to monitor the incident pulse. An unfocused 1.6 MHz broadband transducer was used, driven by a sinusoidal burst of one cycle. Distilled water, castor oil and ethanol have been investigated in a temperature range from 19 to $40^{\circ} \mathrm{C}$. The presented results have been calculated at a frequency of $1.4 \mathrm{MHz}$, and a bias of $\pm 10 \mathrm{~kg} \mathrm{~m}^{-3}$ for reference values from the literature was reported. Furthermore, the apparatus was tested under varying flow conditions and a stable, negative bias of -3 to $-6 \mathrm{~kg} \mathrm{~m}^{-3}$ compared to pycnometer reference measurement was reported. In Adamowski et al. (1995) similar equipment was used and results (average of 15 measurements) of RRM and MRM have been compared for constant temperatures $\left(25 \pm 0.5^{\circ} \mathrm{C}\right)$. In the limited temperature range a bias of $1-2.5 \mathrm{~kg} \mathrm{~m}^{-3}$ could be reached.

Bamberger and Greenwood (2004a, b) and Greenwood and Bamberger (2004) applied the multiple-pulse RRM and used a $5 \mathrm{MHz}$ transducer of $25 \mathrm{~mm}$ diameter. They investigated sodium compound solutions, kaolin slurries and sugarwater solutions. No information about the temperature is 
Table 1. Expectable reflection coefficient difference for a defined density and sound velocity range, different buffer materials and different angles of incidence.

\begin{tabular}{|c|c|c|c|c|c|c|c|}
\hline \multirow[b]{2}{*}{ Material } & \multicolumn{2}{|c|}{ Start value of sample medium } & \multicolumn{2}{|c|}{ End value of sample medium } & \multicolumn{3}{|c|}{ Longitudinal RC difference } \\
\hline & $\begin{array}{c}\rho \\
{\left[\mathrm{kg} \mathrm{m}^{-3}\right]}\end{array}$ & $\begin{array}{c}c \\
{\left[\mathrm{~m} \mathrm{~s}^{-1}\right]}\end{array}$ & $\begin{array}{c}\rho \\
{\left[\mathrm{kg} \mathrm{m}^{-3}\right]}\end{array}$ & $\begin{array}{c}c \\
{\left[\mathrm{~m} \mathrm{~s}^{-1}\right]}\end{array}$ & $\begin{array}{l}\text { angular } \\
\text { incidence }\left(45^{\circ}\right)\end{array}$ & $\begin{array}{l}\text { angular } \\
\text { incidence }\left(25^{\circ}\right)\end{array}$ & $\begin{array}{l}\text { normal } \\
\text { incidence }\end{array}$ \\
\hline PMMA & \multirow{4}{*}{1.055} & \multirow{4}{*}{1510} & \multirow{4}{*}{1.010} & \multirow{4}{*}{1535} & 0.0095 & 0.0111 & 0.0120 \\
\hline quartz glass & & & & & 0.0026 & 0.0037 & 0.0044 \\
\hline aluminium & & & & & 0.0031 & 0.0038 & 0.0042 \\
\hline stainless steel & & & & & 0.0013 & 0.0016 & 0.0018 \\
\hline
\end{tabular}

given, and in terms of validation this does not matter since reference densities have been determined by weighting a known quantity. It would matter, however, if someone wants to consider applicational aspects, e.g. dynamic temperature changes. A bias of $\pm 10 \mathrm{~kg} \mathrm{~m}^{-3}$ is reported for the sodium compound solutions and $\pm 25 \mathrm{~kg} \mathrm{~m}^{-3}$ for the kaolin slurries. In Greenwood and Bamberger (2004) only the error for the acoustic impedance is given, which ranges from $1.8 \%$ to $-1.9 \%$ for a $6.3 \mathrm{~mm}$ pipe wall and from $-0.9 \%$ to $8.7 \%$ for a $3.8 \mathrm{~mm}$ pipe wall. The acoustic velocities have been measured by an independent system. Both the accuracy and the velocity values are not presented. In fact Bamberger and Greenwood presented a validation of the acoustic impedance and not the density. And since the velocity values are missing, an estimation of the density accuracy from the impedance validation data is not possible. There are two quite astonishing facts that are not cleared up in the publication. Table 1 in Greenwood and Bamberger (2004) indicates that only a few certain echo amplitudes are used to analyse the amplitude slope, but it is not stated why not all echoes or why exactly the presented echoes have been chosen. Furthermore, it is stated that the echo slope is a self-calibrating feature to overcome the influence of variations in the excitation voltages. But to prove the stability only the pulse width has been changed, although the published information indicates that the pulser voltage can be varied.

In summary, the following facts can be stated:

- Using the RRM to determine the RC, only buffer reflections are necessary. However, to calculate the density of the sample, the sound velocity in the medium is still necessary. Thus, aside from the angular approach (ARM), at least one echo from a reflector or some additional transmission measurements are required to determine the density.

- The RC of the used reference medium $r_{12}$ (reference) either has to be known or, like in the case of air, can assumed to be equal to 1 .

- The RRM is based on two separate measurements - of the sample and of the reference medium. The assump- tion of similar attenuation $\alpha_{1}$ and $\mathrm{RC} r_{10}$ is only valid if a similar temperature distribution across the buffer can be guaranteed for reference and sample measurement.

- The one-pulse RRM is most susceptible to errors. The assumption of constant excitation pulses is not always valid, and has a great impact on the accuracy of the method. The excitation pulse is practically never exactly the same, and considering ageing of piezoelectric materials, the practical application would need periodic calibrations.

Besides the MRM, dual and multiple pulse RRM which are independent of the excitation amplitude, several alternative strategies have been developed to overcome the problem of varying excitation amplitudes. In Lynnworth and Pedersen (1972), Rychagov et al. (2002) and Jensen (1981) and Deventer (2004) a reference path approach is applied to monitor the excitation variations. The part of the signal that is reflected from a reference interface of constant properties can be used to standardize the received signal and negate excitation variations. Another option is the combination of reference and sample measurement as proposed by Greenwood et al. (1999, 2000) and Guilbert and Sanderson (1996). In this way the same pulse excitation can be sent to reference and sample measurement transducer. Comparable temperature distribution in both buffers can be assumed as well. But using two different transducers probably generates other systematic errors due to misalignment or differing transducer properties. A special case of this method is presented by Püttmer and Hauptmann (1998) and Püttmer et al. (1998, 2000), who used an additional delay line that is connected to the reverse side of the piezoceramic to determine signals from a reference interface. In this way a similar excitation pulse can be guaranteed for reference and sample measurement by using one transducer only. However, the advantage of similar temperature distributions is lost. A clear separation of each pulse is obtained by choosing a different length for the reference buffer and correcting the resulting difference by a calibration factor. In Fisher et al. (1995) a double buffer similar to Deventers MRM was used. However, instead of using the echo of the first buffer to calculate the RC directly, the additional 
reference echo was used to compensate effects such as ageing or depolarization of the piezoceramic.

\subsection{Transmission methods (TM)}

The TM contains all methods that use sender and receiver separately in a parallel assembly to determine the RC. Generally the TM can be classified into two approaches: the first approach is based on the work of Hale (1988), who uses only receiver signals (TMOR); the second approach as presented by Bjørndal et al. (2008) uses the signals of both transducers (TMSR).

Even though Hale's approach is not a true buffer rod technique, it is worth mentioning since it is the basis for further developments. Hale used a transmitter-receiver configuration without any additional delay line. The used configuration and terminology is given in Fig. 4, for which in Hale's approach medium 1 is the sender and medium 3 is the receiver.

Hale assumed that the attenuation does not change significantly for fluids of quite similar composition (like tap water and salty water) and that the sender impedance equals the receiver impedance $\left(Z_{1}=Z_{3}\right)$. Therefore, it was possible to state that any change in acoustic impedance of the sample liquid $Z_{2}$ is directly proportional to the measured change of amplitude $A_{4}$ :

$A_{1}=\frac{\left(Z_{1}+Z_{2}\right)^{2}}{4 e^{-\alpha_{2} l_{2}} Z_{1} Z_{2}} A_{4}$.

Considering calibration measurement for two liquids (indices $\mathrm{c} 1$ and c2) of known acoustic impedances $Z_{\mathrm{c} 1}$ and $Z_{\mathrm{c} 2}$ and constant excitation amplitude $A_{1}$, one reaches

$\frac{\left(Z_{1}+Z_{\mathrm{c} 1}\right)^{2}}{4 \exp \left(-\alpha_{\mathrm{c} 1} l_{2}\right) Z_{1} Z_{\mathrm{c} 1}} A_{4 \mathrm{c} 1}=\frac{\left(Z_{1}+Z_{\mathrm{c} 2}\right)^{2}}{4 \exp \left(-\alpha_{\mathrm{c} 2} l_{2}\right) Z_{1} Z_{\mathrm{c} 2}} A_{4 \mathrm{c} 2}$.

Under the assumption of similar internal losses $\left(\alpha_{\mathrm{c} 1}=\alpha_{\mathrm{c} 2}\right)$ the attenuation term can be neglected, and the impedance $Z_{1}$ can be calculated:

$Z_{1}=\frac{Z_{\mathrm{c} 1}-k Z_{\mathrm{c} 2}}{1-k}+\sqrt{\left(-\frac{Z_{\mathrm{c} 1}-k Z_{\mathrm{c} 2}}{1-k}\right)^{2}-\frac{Z_{\mathrm{c} 1}^{2}-k Z_{\mathrm{c} 2}^{2}}{1-k}}$

where

$k=\frac{\exp \left(-\alpha_{\mathrm{c} 1} l_{2}\right) Z_{\mathrm{c} 1} A_{4 c 2}}{\exp \left(-\alpha_{\mathrm{c} 2} l_{2}\right) Z_{\mathrm{c} 2} A_{4 \mathrm{c} 1}}$

The density results showed less than $2 \%$ variation from the true values which have been determined via weight measurements of known volumes. McGregor (1989) discussed several possible methods to measure the density by using the same probe arrangement like Hale. He stated that a continuous wave system, with and without interference, would provide the most accurate means of determining the velocity and the characteristic impedance of the fluid under test.

Henning et al. (2000) mounted the transducers on a glass tube wall of half-wave thickness. Furthermore, the setup was

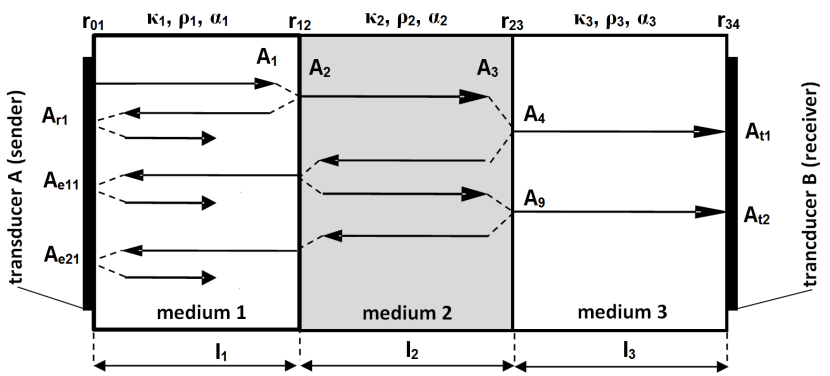

Figure 4. Schema showing the basic principles and relevant pulses for the TM and giving the terminology for Hale's, Henning's and Bjørndal's approach.

calibrated for two liquids of known acoustic impedance to determine $Z_{1}$. But in the case of Henning's setup, $Z_{1}$ is only the apparent transducer impedance. Indeed, this fictive impedance describes the combined impedance of glass wall and transducer as a result of the sound propagation through the glass wall of half-wave thickness. Furthermore the basic TMOR approach was expanded for the amplitude $A_{9}$ :

$\frac{A_{9}}{A_{4}}=\left(\frac{Z_{1}-Z_{2}}{Z_{1}+Z_{2}}\right)^{2} \exp \left(-2 \alpha_{2} l_{2}\right)$.

Still the attenuation is neglected in order to calculate the transducer impedance. But now two equations can be used to calibrate the transducer impedance. Using both Eqs. (32) and (33) a mismatch between the transducer impedances was reported. In the end both impedances have been used to determine the acoustic impedance of the sample liquid. Even though the glass tube wall is of half-wave thickness, it is quite clear from theory that the amplitudes $A_{4}$ and $A_{9}$ as described by the equations are not equal to the amplitudes received by the transducer. From the physical point of view the received pulses are also influenced by the wall material and contain also information from superpositioned reflections inside the tube wall. Nevertheless, in Henning et al. (2000) both the basic and the expanded TMOR have been compared for several liquids using an aerometer measurement as reference. While the basic TMOR showed a bias of 3 to $-40 \mathrm{~kg} \mathrm{~m}^{-3}$, the expanded TMOR resulted in a bias of -16 to $10 \mathrm{~kg} \mathrm{~m}^{-3}$. Furthermore, it was reported that the absolute error increases to a few percent in the case of increasing sound absorption corresponding to the liquid properties or diffuse scattering at particles.

Additionally to the signals of the receiver (transducer B), Bjørndal et al. (2008) employs pulses received by transducer A. Comparable with the MRM, one achieves an equation that cancels the influence of the attenuation, the transducer and the electronics sensitivity. Bjørndal employs two pulses of transducer A and two pulses of transducer B (R_echo12_12 method, terminology given in Fig. 4):

$r_{12}= \pm\left(1-\frac{A_{\mathrm{e} 11} A_{\mathrm{t} 1}}{A_{\mathrm{r} 1} A_{\mathrm{t} 2}}\right)^{-0.5}$. 
It is reported that the systematic deviation from reference values was slightly higher for the TMSR compared with MRM, and it is stated that using information of both transducers, non-identical sound fields and a misalignment in the transducer configuration might be the reason for the higher deviation. In Bjørndal and Frøysa (2008) all possible pulse combinations besides Eq. (34) are discussed, even some further methods that employ transmitted pulses from both sides in which transducer A and B are used alternately as senders. After a detailed uncertainty analysis with respect to bit resolution and noise, it was outlined that the R_echo12_12 method (Eq. 34) possesses a relative uncertainty close to the optimal and case-dependent R_echo123_123 (which uses 3 pulses of receiver and transducer; details in Bjørndal and Frøysa (2008) and may be the best choice of all TMSR to be compared with the MRM).

\subsection{Angular reflection method (ARM)}

The ARM was presented first by Greenwood and Bamberger (2002) and Greenwood et al. (1999). Concerning the determination of the RC, the ARM is a simple one-pulse RRM (Eq. 24). But to determine the sound velocity and the density of the medium (see Eq. 5) the ARM uses measurements at two different angles.

The RC of the longitudinal wave, $r_{\mathrm{LL}}$ at a given angle of incidence (see Fig. 5) depends on the angle $\beta_{\mathrm{L}}$, the density $\rho$, the longitudinal velocity $c$ of the sample liquid and the longitudinal velocity $c_{\mathrm{L}}$, the shear velocity $c_{\mathrm{T}}$ and the density $\rho_{\mathrm{S}}$ of the buffer material (Greenwood et al., 1999; Krautkramer and Krautkramer, 1983). The equations are generally given as

$r_{\mathrm{LL}}=\frac{G-H+J}{G+H+J}$,

where

$G=\left(\frac{c_{\mathrm{T}}}{c_{\mathrm{L}}}\right)^{2} \sin 2 \beta_{\mathrm{L}} \sin 2 \beta_{\mathrm{T}}$,

$H=\cos ^{2} 2 \beta_{\mathrm{T}}$,

$J=\frac{\rho c \cos \beta_{\mathrm{L}}}{\rho_{\mathrm{S}} c_{\mathrm{L}} \cos \beta}=\frac{Z_{2} \cos \beta_{\mathrm{L}}}{Z_{1} \cos \beta}$,

and from Snell's law,

$\sin \beta=\frac{c \sin \beta_{\mathrm{L}}}{c_{\mathrm{L}}}, \sin \beta_{\mathrm{T}}=\frac{c_{\mathrm{T}} \sin \beta_{\mathrm{L}}}{c_{\mathrm{L}}}$.

Instead of measuring the sound velocity $c$, the RC is determined using an RRM approach (Eq. 24) to calculate the parameter $J$ via Eq. 35). Now Eqs. (38) and (39) can substitute the unknown angle $\beta$ in

$\sin ^{2} \beta+\cos ^{2} \beta=1$.

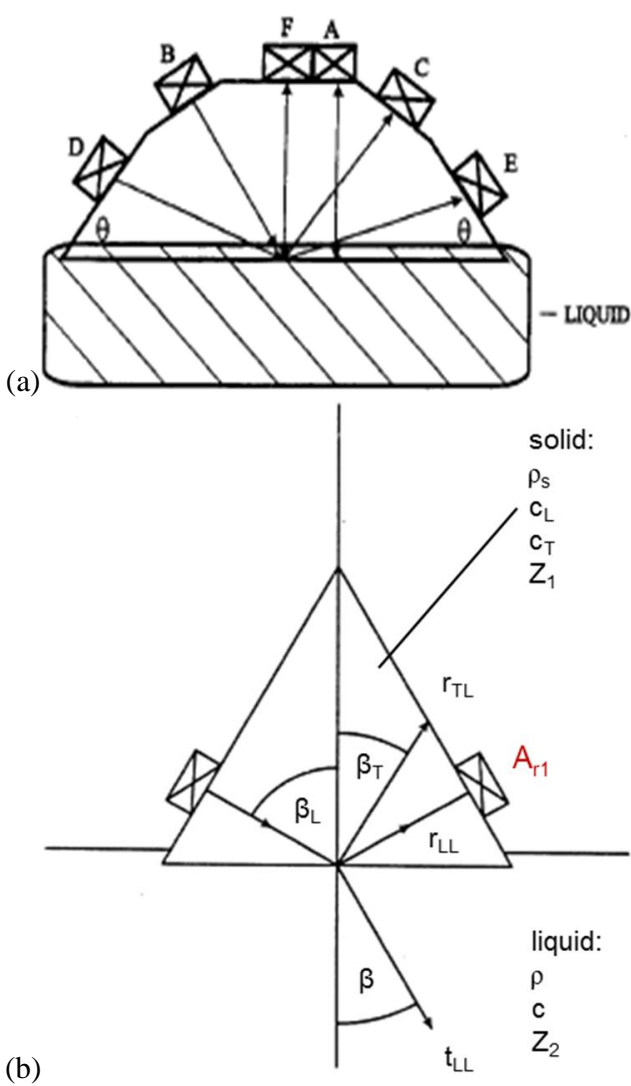

Figure 5. Schematic showing (a) the wedge design of Greenwood and Bamberger, (b) the design given by Krautkramer and the definitions of terminology.

Doing so for two different angles, equalizing both and writing the resulting equation in terms of $\rho$ gives a term which is independent from the sound velocity in the liquid:

$\rho=\rho_{\mathrm{S}}\left(\frac{\sin ^{2} \beta_{\mathrm{L} 1}-\sin ^{2} \beta_{\mathrm{L} 2}}{\cos ^{2} \beta_{\mathrm{L} 1} / J_{1}^{2}-\cos ^{2} \beta_{\mathrm{L} 2} / J_{2}^{2}}\right)^{0.5}$.

Finally, the sound velocity in the liquid can be calculated with

$c=\left(\frac{\sin ^{2} \beta_{\mathrm{L}}}{c_{\mathrm{L}}^{2}}+\frac{\rho^{2} \cos ^{2} \beta_{\mathrm{L}}}{J^{2} Z_{1}^{2}}\right)^{-0.5}$

In summary the following facts can be stated:

- The great advantage of the ARM is the determination of the sound velocity on the basis of reflection coefficient measurements at two angles. Only signal information from the interface is required, and therefore no sound propagates through the sample medium.

- The basics of the ARM reflection coefficient determination are comparable to the RRM. Consequently, all facts stated for the RRM also count for the ARM. Only the sound velocity determination is different. 
- The ARM also provides the opportunity to measure the sound velocity via pulse-echo or transmission approach. Instead of measurements at two angles, one would be sufficient. The missing angle $\beta$ in Eq. (38) could be calculated via Eq. (39).

- The angle and the temperature-dependent parameters density, longitudinal and transversal sound velocity of the buffer material have to be known precisely. The slightest deviation from the real value can generate a significant error in the density.

The ARM was validated for sugar-water solutions and surrogate slurries via weighting of known volumes. For the analysis of the sugar-water samples the wedge was submerged to reach a uniform wedge temperature. An error of $0.1-1.3 \%$ was reported, which is a bias of $1-14 \mathrm{~kg} \mathrm{~m}^{-3}$. The experiments for the surrogate slurries have been accomplished at a test loop for varying slurry flow rates, aeration flow rates and two constant temperatures $\left(25\right.$ and $\left.50{ }^{\circ} \mathrm{C}\right)$. Each density was calculated by averaging 45 signals. The validation was accomplished by comparing the average of 40 sensor densities with reference densities. The bias varied between 13 and $260 \mathrm{~kg} \mathrm{~m}^{-3}$. Neglecting some extreme deviations, an overall bias of $20 \mathrm{~kg} \mathrm{~m}^{-3}$ could be accomplished.

\section{Probe design considerations}

The design of ultrasonic density probes as presented by the aforementioned authors is a complex process. In most publications, the probe's dimensions and material are simply mentioned as a given fact, not as a required necessity. In fact, an unequivocal identification of clearly unaffected pulses is one of the basic requirements for all presented methods. As soon as one of the required pulses is superpositioned by any other pulse or effect, which is not considered by the plane wave propagation theory, the resulting values will be affected by a systematic error.

\subsection{Pulse excitation and separation}

The best way to exemplify all interrelations clearly is to follow the design process of a buffer which might be used for an RRM approach. In its simplest version, we want to see the first reflected pulse, only affected by the reflection at the interface and the buffer material's attenuation. Neglecting all application-based boundary conditions, the only real limiting conditions are the choice of the ultrasound source and the frequency of and the type of excitation pulse. By making the right choice one can affect the pulse duration. Choosing a transducer which generates a low-damped narrowband pulse of low frequency, one achieves a relatively long pulse. Choosing a high frequency, highly damped broadband pulse, one achieves a short pulse. If a burst excitation of several cycles is used, one can specify the frequency quite accurately, but this generates a long-lasting sound pulse. Using a pulse excitation, one can generate a shorter sound pulse, but the pulse frequency generally relies on the system's resonance frequency. In any case, often the most convenient way to investigate the resulting sound pulse duration is to test and measure the pulse length $t_{\mathrm{p}}$ of a chosen ultrasound source for varying excitation pulse amplitudes, cycles and frequencies. Knowing $t_{\mathrm{p}}$ and the temperature-dependent sound velocity $c_{1}$ of the buffer material, it is possible to calculate the minimum buffer thickness for a given temperature range to prevent superposition phenomena for the multiple buffer reflections $A_{\text {rn }}$.

When a reflector is used to determine the sound velocity or to adopt the MRM, further parameters besides the temporal determination of the pulse position are relevant to prevent superposition of buffer reflections and echoes. If so, the pulse amplitude and the amount of buffer reflections also have to be considered. For constant excitation amplitude those parameters only depend on the buffer materials absorption and the RC at the interphase. Combined with the pulse length $t_{\mathrm{p}}$ those parameters define the buffer reflections duration $t_{\mathrm{br}}$. In order to prevent superposition between the buffer reflections $A_{\text {rn }}$ and the echo pulses $A_{\mathrm{e} j k}$, the following condition has to be fulfilled:

$\frac{l_{2}}{c_{2}}=\mathrm{TOF}_{2}>t_{\mathrm{br}}$,

where $\mathrm{TOF}_{2}$ is the signal's time of flight in the sample medium. Alternatively, dimensions and materials can be designed in a way that the echo pulses arrive in a time gap between two buffer reflections. This target is hard to achieve since the echo position depends on the sample mediums sound velocity, and thus such special designs are often usable only for a defined sample medium and temperature range (Bjørndal et al., 2008; Bjørndal and Frøysa, 2008). In the case of the MRM as introduced by Papadakis the superposition between the 1 st pulses of the 1 st and 2 nd echo $\left(A_{\mathrm{e} 11}\right.$ and $\left.A_{\mathrm{e} 21}\right)$ and the reflections of those pulses inside the reflector have to be eliminated, and then the condition $l_{3} / c_{3}=\mathrm{TOF}_{3}>t_{\mathrm{p}}$ is satisfied. Bjørndal et al. (2008) presents most of those dimensional considerations. Additionally, Bjørndal and Püttmer (1998) introduce conditions for edge wave contributions with and without mode conversion. The edge wave distributions mainly depend on the buffer diameter and the ratio of transducer radius to buffer thickness and therefore also represent the near-field phenomena. The mode conversion depends on the shear wave velocity and therefore on the elastic properties of the buffer material.

\subsection{The choice of material}

As indicated in the previous section, most design considerations depend on the material's properties. Thus, besides the option to change the dimension of buffer or reflector, one can simply change the material to achieve a desired signal pattern. The choice of material also defines the resolution that 
has to be reached for a given process of defined density range. The following table shows start and end values (density and sound velocity) of a typical yeast fermentation and the resulting $\mathrm{RC}$ difference that can be expected for different buffer materials.

Indeed, it becomes apparent that according to Eq. (9) any buffer material can be used to determine the density using the reflection coefficient. But, as shown in Table 1, only materials of acoustic impedance comparable to the impedance of the sample medium possess an acceptable sensitivity for small density variations (Püttmer and Hauptmann, 1998; Püttmer et al., 2000; Bjørndal et al., 2008; Greenwood et al., 1999). The same holds true for the ARM; increasing angular difference to the normal incidence even decreases the RC difference.

Additional requirements for the buffer materials are good chemical resistance, reasonable temperature stability and a low sound attenuation (Püttmer and Hauptmann, 1998; Püttmer et al., 2000). If special liquids are analysed, e.g. suspensions containing abrasive materials, further criteria such as mechanical resistivity may be of importance. Concerning the mode conversion in the case of angular incidence - for example, if the ARM is applied or in the case of edge waves - the elastic properties of the buffer material may also be of interest. Materials of a high Poisson's ratio generally possess a higher conversion to shear waves.

Besides deploying the choice of material to guarantee a clear pulse separation, the pulse amplitude can be affected. Choosing a buffer material of acoustic impedance, comparable with the sample mediums impedance, results in a low reflection coefficient. The buffer reflections $A_{\mathrm{rn}}$ are less in quantity and lower in amplitude. Most of the energy is transferred into the sample medium. However, if an echo comes back $\left(A_{\mathrm{e} 11}\right)$, most of the energy is transferred back into the buffer. Thus probably too little energy remains for a second detectable echo $\left(A_{\mathrm{e} 21}\right)$. The same holds true for the reflector. Choosing a reflector material of high acoustic impedance results in high echo amplitudes. However, materials of high acoustic impedance generally possess high sound velocity, low sound attenuation and a high reflection coefficient. Therefore, resulting from extensive reflector dimensions and a considerable amount of reflections inside the reflector, this may interfere with the second echo $\left(A_{\mathrm{e} 21}\right)$. In such cases a special reflector shape often is the most feasible alternative (Carlson et al., 2003a; Deventer and Delsing, 2001b). A reflector of low acoustic impedance may simplify the task to achieve the maximum signal purity, but also results in lower echo amplitudes.

\subsection{Temperature variation, sound field and signal-to- noise ratio considerations}

Regardless of the method applied or material chosen, if the temperature changes, everything changes concerning sound propagation. This fact also counts for design considerations. Every single boundary condition mentioned above has to be valid for the entire temperature range. If the temperature changes, so does the speed of sound, density, sound absorption and dimensions of all materials involved. Therefore, not only does the pulse's position change but also the pulse amplitudes. In the best-case scenario, the amplitude slightly decreases; in the worst case, whole pulses are no longer detectable, which might hamper the analysis of RC or ultrasound velocity (USV). Mak (1991) compared several MRMs concerning the influence of systematic (beam diffraction) and random errors (noise). He showed that varying attenuation and signal-to-noise ratio (SNR) affect the method's error. The higher the SNR and the less influence of diffraction, the smaller the errors in the RC. Therefore, the reference methods (ARM, RRM) might show better results, since they are independent from beam diffraction, while the accuracy of the MRM depends on the accuracy of the diffraction correction. Mak used a $50 \mathrm{MHz}$ broadband transducer. Both the reference methods and the MRM showed quite low RCs at low frequencies, and both methods converged for higher frequencies near the transducer's centre frequency and showed comparable results. Adamowski et al. $(1995,1998)$ used a constructive solution to eliminate diffraction issues. The so-called DET technique employs a receiver of an aperture larger than the emitter that generates the sound field. As long as the beam spreading does not reach the dimensions of the receiver diameter, the principles of MRM for plane wave propagation are valid without correction.

While the correction of diffraction in the far field is discussed by several authors (Papadakis, 1959; Papadakis et al., 1973; Bjørndal et al., 2008; Kushibiki et al., 2003), the near-field problem is often not mentioned at all. Although the beam is assumed to be parallel in the near field (Povey and McClements), it is recommended to avoid it totally. The intensity varies greatly with distance, the surface's amplitudes are not constant and the whole wave front cannot be expected to be normal to the phase velocity vector. Essentially the plane wave propagation is not valid within the near field. Consequently, besides all dimensional considerations mentioned in Sects. 3.1 and 3.2, the first condition that has to be kept is the near-field distance $N$ between the sender and first interface:

$N=\frac{a^{2}}{\lambda}$,

with a being the transducer radius. Table 2 shows methodic details as applied by different authors and the resulting nearfield length in comparison to the chosen buffer length. Besides Greenwood, who applied the ARM, and Papadakis, who applied the MRM for attenuation measurements, the researchers used the path length of dimensions (double buffer rod length) greater than or at least in the range of the nearfield distance.

Diffraction effects are generally corrected via Williams' expression (Williams, 1951; Williams and Labaw, 1945). Although Williams stated that his expression is only accurate 
Table 2. Near-field relevant, methodic details of relevant publications.

\begin{tabular}{|c|c|c|c|c|c|}
\hline Source & $\begin{array}{l}\text { Transducer } \\
\text { diameter } d[\mathrm{~mm}]\end{array}$ & $\begin{array}{l}\text { Centre } \\
\text { frequency } f[\mathrm{MHz}]\end{array}$ & Material & $\begin{array}{c}\text { Buffer rod } \\
\text { length }[\mathrm{mm}]\end{array}$ & $\begin{array}{l}\text { Near-field } \\
\text { distance } N[\mathrm{~mm}]\end{array}$ \\
\hline Adamowski et al. $(1995,1998)$ & 19.0 & 1.6 & PMMA & $30.0 \quad 42.0$ & 53.48 \\
\hline Bjørndal et al. (2008) & 12.5 & 5.0 & aluminium & 80.0 & 30.90 \\
\hline Deventer and Delsing (1997) & 10.0 & 3.7 & PEEK/PMMA & $26.0 \quad 20.0$ & 34.26 \\
\hline Greenwood et al. (1999) & 12.5 & 2.25 & Rexolite & 6.3 & 37.56 \\
\hline McClements and Fairly $(1991,1992)$ & 10.0 & 2.1 & PMMA & 40.0 & 19.44 \\
\hline Papadakis (1968) & 12.7 & 10.0 & $\begin{array}{l}\text { fused quartz } \\
\text { aluminium } \\
\text { steel }\end{array}$ & $\begin{array}{c}25.4 / 62.2 \\
25.4 \\
18.9\end{array}$ & $\begin{array}{l}67.66 \\
63.20 \\
68.34\end{array}$ \\
\hline Püttmer and Hauptmann (1998) & 20.0 & 2.0 & quartz glass & 31.0 & 33.67 \\
\hline
\end{tabular}

for $k \cdot a>100$ and distances $z_{\mathrm{W}} \geq\left(k \cdot a^{4}\right)^{1 / 3}$, the exact expression without approximations (see Williams, 1951, Eq. 17) might be usable in an extended domain. Nevertheless, so far it has not been reported whether corrections in the near field or for sound fields across an interphase within the near field can be applied successfully to reach a reflection coefficient accuracy of 1E-4 or less (see Table 5).

Knowing all these facts it becomes clear that if spatial limitations for the sensor application exist and a buffer miniaturization becomes necessary, only increasing the pulse frequency to achieve pure signals is not enough. Often the dimensions of the transducer with respect to the buffer mediums sound velocity have to be adapted.

\subsection{Constructional uncertainties}

The main constructional uncertainty which is occasionally discussed is the parallelism of surfaces. In ARMs, of course, the accuracy of the angles will be of similar importance. In Carlson et al. (2003b) it is reported that the misalignment of the transducer to buffer material is the main source of error causing an overestimation of attenuation and acoustic impedance. In Bjørndal et al. (2008) it is stated that effects of nonparallelism can be neglected for surfaces that are parallel within $0.01 \mathrm{~mm}$. In Adamowski et al. (1995) a maximum parallelism of $0.0004 \mathrm{~mm} \mathrm{~mm}^{-1}$ and a change of $0.7 \%$ in the reflection coefficient for an intentionally caused misalignment of $0.0024 \mathrm{~mm} \mathrm{~m}^{-1}$ was reported.

\section{Discussion}

While reviewing critically all published methods and validation results with regard to validation complexity, error analysis and real process relevance, several gaps and questions appeared which will be discussed in the following sections. The first point will be the analysis of relevant pulses. Further points will include the equipment used for ultrasound generation and detection, reference density and temperature measurement, the sound velocity determination and extended uncertainty considerations.

\subsection{Signal processing}

Signal processing is a wide field with many fundamental details. The applied methods range from simple time domain (Greenwood and Bamberger, 2002; Greenwood et al., 1999) to extensive frequency domain methods (Bjørndal et al., 2008). The equations presented so far represent the time domain approach and refer to the signal amplitude, but do not state which pulse amplitude is used in the end. In Greenwood and Bamberger (2002), Greenwood et al. (1999), Püttmer and Hauptmann, (1998) and Püttmer et al. (1998, 2000), the maximum peak-to-peak amplitude within a certain time window has been examined:

$A_{\text {pulse }}=\operatorname{maximum}\left[A\left(t_{\mathrm{w} 1}: t_{\mathrm{w} 2}\right)\right]-\operatorname{minimum}\left[A\left(t_{\mathrm{w} 1}: t_{\mathrm{w} 2}\right)\right]$,

where $A_{\text {pulse }}$ represents the value that is inserted in the respective equation of reflection coefficient calculation and $t_{\mathrm{w} 1}$ and $t_{\mathrm{w} 2}$ the time boundaries of the analysed pulse. In the following sections, $A(t)$ will represent the pulse in the time domain and $a(f)$ in the frequency domain.

Papadakis (1968) had started analysing amplitudes in the time domain for attenuation analysis, but later he changed to spectrum analysis (Papadakis et al., 1973). After correcting the frequency dependent diffraction, Papadakis et al. analysed the frequency-dependent reflection coefficient and attenuation (Papadakis et al., 1973; Sachse, 1974):

$A_{\text {pulse }}(f)=a(f)$.

It was found (Sachse, 1974) that the reflection coefficient and density are nearly constant over a frequency range around the centre frequency of the transducer's maximum response. That might be the reason for obtaining the amplitudes from the spectra at a particular frequency $\left(f_{1}\right)$ (Adamowski et al., 1995). Higuti (Higuti and Adamowski, 2002b; Higuti et al., 2001), who followed the DET approach of Adamowski, introduced the energy method, in which the energy spectral 
density of each pulse is used for the reflection coefficient analysis:

$A_{\text {pulse }}=\int_{-\infty}^{+\infty}|a(f)| \mathrm{d} f$.

It is stated that the deployment of the energy method results in smaller variations when compared to the singlefrequency method, because it averages the noise over frequency. For added Gaussian white noise of varying amplitude to simulation results, Higuti found that the energy method improves the results with smaller SNRs. By calculating the spectral density only for a small frequency band, the performance could be enhanced due to the rejection of frequencies outside the band of the transducer. Experimental results showed an error of less than $0.2 \%$ and proved the enhanced performance of the presented new signal processing method.

In Bjørndal et al. (2008) a more detailed analysis of signalprocessing methods in the time and frequency domain is presented. In the time domain the amplitude value was not determined simply as the main peak-to-peak difference per pulse; instead the peak-to-peak value was determined per period:

$A_{\text {pulse }}=\operatorname{maximum}\left[A(t)_{\mathrm{pn}}\right]-\operatorname{minimum}\left[A(t)_{\mathrm{pn}}\right]$,

where $A(t)_{\mathrm{pn}}$ represents the $n$-th period of the analysed time domain pulse. Depending on the amount of analysed periods (e.g. from $P_{1}$ to $P_{2}$ ) one can calculate a mean reflection coefficient $R_{\mathrm{m}}$ for each signal (Bjørndal et al., 2008):

$R_{\mathrm{m}}=\frac{1}{P_{2}-P_{1}+1} \sum_{n=P_{1}}^{P_{2}} R_{n}$.

It is reported (Bjørndal et al., 2008) that if the first period of the waveforms is included, there may be large errors, particularly when the amplitudes are analysed in the time domain, but also in the case of the frequency domain analysis. In the frequency domain the analysis followed the spectral density approach (Eq. 47), but the so-called 12 norm was introduced based on the mathematic basics of $L^{\mathrm{p}}$ spaces:

$A_{\text {pulse }}=\sqrt{\int_{f_{1}}^{f_{2}}|a(f)|^{2} \mathrm{~d} f}$.

It is stated (Bjørndal et al., 2008) that the frequency domain integration introduces a spectral-averaging approach, reducing the effect of single-frequency interference in the echo signals. The 12 norm accentuates the dominant part of the frequency spectrum, making it easier to evaluate the effect of the upper frequency limit. Equally to the periodic peak-to-peak analysis in the time domain, the frequency spectrum was analysed on a half-periodical basis. Additionally, a Hanning window function was applied to reduce the spectral leakage. The windows have been centred at the local extreme values of each analysed peak (Bjørndal et al., 2008). The accuracy improvement compared to a frequency domain approach without window function was not reported.

Applying the different signal-processing methods to PSPICE simulation results, it was found (Bjørndal et al., 2008) that the frequency domain approach gives significantly less density deviation than the time domain analysis. The experimental results could not confirm the theoretical evaluation; in some cases the time domain analysis indicates more accurate results and less deviation. Furthermore, Bjørndal suggested a time domain integration method following Raum et al. (1998), but it was also adverted to the high sensitivity of the time integration approach to DC offsets and waveform disturbance effects:

$A_{\text {pulse }}=\int_{t_{1}}^{t_{2}}|\operatorname{env}(A(t))| \mathrm{d} t$.

Besides the different signal analysis methods, the signalprocessing parameters and the applied preprocessing steps are of high relevance to reach the reported accuracies. Concerning the preprocessing, most authors mentioned that a certain amount of signals have been averaged before applying the different signal analysis methods. Through signal averaging the SNR can be enhanced and the amplitude resolution can be increased beyond the AD-converter limitations (Bjørndal et al., 2008). The use of a $25 \mathrm{MHz}$ low-pass filter is mentioned in Bjørndal et al. (2008); further references for filter usage have not been found. Furthermore, in Bjørndal et al. (2008) the use of least-squares-sense cubic spline approximation was reported to increase the vertical and temporal resolution.

Relevant signal-processing parameters are the pulse length in time, the amount of data points with respect to the sampling rate, the amplitude resolution and the usage of any additional processing steps to improve the frequency or magnitude accuracy, such as filtering, signal averaging, zero padding or application of window functions. Table 3 overviews the signal-processing details of several relevant authors with regard to the reached accuracies.

\subsection{Signal generation and detection}

Most authors used highly advanced equipment for their investigations. Generally pulse or function generators provide the electrical pulse which is converted to sound pulses by commercially available transducers. After amplification, the signal is recorded by an oscilloscope and conveyed to a personal computer for further signal analysis. Standard signal generators are generally limited to $20 \mathrm{~V}$ peak excitation, which is sufficient for most of the investigations. Custom signal generators for higher excitation voltages and amplifiers are available but require special circuits since the input voltage of commercial oscilloscope is often limited. To avoid noisy interferences and overloading of the oscilloscope, the 
Table 3. Processing details from different literature sources with regard to density accuracies.

\begin{tabular}{|c|c|c|c|c|c|c|}
\hline Source & $\begin{array}{l}\text { Window } \\
\text { size }\end{array}$ & $\begin{array}{r}\text { Sampling } \\
\text { rate }(\mathrm{MHz})\end{array}$ & $\begin{array}{r}\text { Averaged } \\
\text { signals }\end{array}$ & $\begin{array}{l}\text { Applied } \\
\text { method }\end{array}$ & $\begin{array}{l}\text { Used } \\
\text { domain }\end{array}$ & $\begin{array}{l}\text { Density } \\
\text { accuracy }\end{array}$ \\
\hline Adamowski et al. $(1995,1998)$ & 500 (1024, zero padding) & 100 & 64 & MRM & time/frequency & $1.50 \%$ \\
\hline Bjørndal et al. (2008) & 1000 (32 768, zero padding) & 59 & 256 & MRM, TRM & time/frequency & $0.15 \%$ \\
\hline Deventer and Delsing (1997) & 512 & 200 & 32 & MRM & frequency & $<1 \%$ \\
\hline Greenwood et al. (1999) & 4096 & 40 & 45 & ARM & time & $<1 \%$ \\
\hline Bamberger and Greenwood $(2004 a, b)$ & - & - & - & RRM & frequency & $<1 \%$ \\
\hline McClements and Fairly $(1991,1992)$ & - & 100 & $\approx 2000$ & RRM & time/frequency & $0.50 \%$ \\
\hline $\begin{array}{l}\text { Papadakis et al. (1973), } \\
\text { Papadakis (1968) }\end{array}$ & - & - & - & MRM & time/frequency & - \\
\hline $\begin{array}{l}\text { Püttmer and Hauptmann (1998), } \\
\text { Püttmer et al. (2000) }\end{array}$ & - & - & - & RRM & time & $0.20 \%$ \\
\hline
\end{tabular}

excitation and receiving circuit should be decoupled. Results concerning the influence of excitation voltage and voltage variations on the methods accuracy are not reported. While in Greenwood and Bamberger (2004) it is stated that the decay RRM approach is independent of changes in the pulser voltage, and although it can be assumed that the MRM is independent from the excitation voltage, it is quite doubtful that the density error is totally independent. A change of the excitation voltage or signal amplification might change the degree of interference between subsequent pulses, the SNR and the pulse appearance. The independency has definitely not been proven experimentally so far. The same counts for the excitation and transducer type. Results are reported for different excitation types (Table 4 shows an overview) ranging from peak, rectangular and sinusoidal pulses to bursts of several cycles, but a decent comparative evaluation is missing so far. Indeed, in Bjørndal et al. (2008) simulation results are reported for varying cycles, but a comparison to peak excitation and an experimental evaluation were not shown. Moreover, investigations regarding the transducers type or piezoelectric materials (PEM) have not been found so far. It is known that the very different properties of the PEM result in completely different probe types (Lach et al., 1996). Concerning the determination of the reflection coefficient, different transducers constructed with different PEM might show different sensitivities and variance.

Concerning measurements in real process environments, the use of general purpose equipment, such as oscilloscopes or function generators, is a double-edged sword. Indeed it is commercially available technology of proven accuracy, but it is often both immoderate and unfeasible for specific tasks such as reflection coefficient determination. Using the typical sampling frequency of $250 \mathrm{MHz}$ to characterize a $2 \mathrm{MHz}$ signal in the frequency domain is clearly oversampling - no additional information is extracted, but it might be necessary to reach high time of flight or amplitude accuracy in the time domain. In the end, the effort for signal-processing increases dramatically with increasing sampling frequency. Indeed, standard oscilloscopes can monitor the voltage-time course with a high sampling frequency but provide only a moderate vertical resolution of 8 bit. Based on simulation results it was shown (Püttmer et al., 2000; Bjørndal and Frøysa, 2008; Bjørndal et al., 2008) that a 12-bit resolution is the best choice to reach reasonable errors. Since the price of an oscilloscope is not negligible, the vertical resolution is quite low and no further usable features like amplification or variable programmable signal processing steps are provided, an oscilloscope often is replaceable. As shown in Greenwood et al. (1999, 2006), a time-to-digital converter with reasonable sampling frequency and an analogue-to-digital converter with reasonable vertical resolution also serve the purpose. Similar considerations apply to signal generation and processing. An arbitrary function generator and a personal computer might not be the best choice for measurements in real process environments, but as long as it is not clear which excitation function is the best choice for a certain method, reports about compact units that incorporate all main tasks, signal generation, signal detection and signal processing will take a while in coming.

\subsection{Reference analytics, validation and uncertainty considerations}

The following section reviews and discusses the measurement uncertainties in terms of density determination via BRT of all significantly involved variables: density, reflection coefficient, ultrasound velocity and temperature.

Besides the uncertainties of the simplification in Eq. (14) the reflection coefficient mainly depends on the amplitude error. According to the propagation of uncertainty the degree of dependency is defined by the equation of each method (Eqs. 19, 24, 27, 29 and 34). The amplitude error basically depends upon three main factors: the amplitude resolution, the time resolution and the SNR. The amplitude resolution dependency was discussed in Bjørndal and Frøysa (2008), Bjørndal et al. (2008) and Püttmer et al. (2000); both research groups arrived at the conclusion that a resolution of 12 bit or better is required to reach accuracies below $0.5 \%$ error. 
Table 4. Details of sound generation equipment as published by different authors.

\begin{tabular}{|c|c|c|c|c|c|}
\hline \multirow{2}{*}{$\begin{array}{l}\text { Author/Source } \\
\text { Adamowski et al. (1995, } \\
\text { 1998) }\end{array}$} & \multicolumn{2}{|c|}{ Equipment } & \multicolumn{2}{|c|}{ Excitation } & \multirow{2}{*}{$\begin{array}{l}\text { Transducer } \\
\text { KB-Aerotech }(1.6 \mathrm{MHz})\end{array}$} \\
\hline & $\begin{array}{l}\text { function } \\
\text { generator }\end{array}$ & oscilloscope ( 8 bit) & pulse/ burst & $2-3$ cycles & \\
\hline Bjørndal et al. (2008) & $\begin{array}{l}\text { function } \\
\text { generator }\end{array}$ & oscilloscope ( 8 bit) & sinusoidal bu & & Panametrics (5 MHz) \\
\hline $\begin{array}{l}\text { Deventer and Delsing } \\
\text { (1997) }\end{array}$ & $\begin{array}{l}\text { pulse } \\
\text { generator }\end{array}$ & oscilloscope ( 8 bit) & pulse & - & Panametrics (5 \& $10 \mathrm{MHz})$ \\
\hline Greenwood et al. (1999) & $\begin{array}{l}\text { function } \\
\text { generator }\end{array}$ & $\begin{array}{l}\text { data acquisition } \\
\text { card (PC)/digitizer } \\
(12 \text { bit })\end{array}$ & burst & 10 cycles & - \\
\hline $\begin{array}{l}\text { Bamberger and Greenwood } \\
(2004 a, b)\end{array}$ & $\begin{array}{l}\text { ultrasonic } \\
\text { pulser }\end{array}$ & oscilloscope (-) & - & & - \\
\hline $\begin{array}{l}\text { McClements and Fairly } \\
(1991,1992)\end{array}$ & $\begin{array}{l}\text { function } \\
\text { generator }\end{array}$ & oscilloscope (-) & burst & $5-10$ cycles & $\begin{array}{l}\text { Karl Deutsch }(0.3-1 \mathrm{MHz}) \text {, } \\
\text { Sonatest }(1-6 \mathrm{MHz})\end{array}$ \\
\hline $\begin{array}{l}\text { Papadakis et al. (1973); } \\
\text { Papadakis (1968) }\end{array}$ & $\begin{array}{l}\text { pulse } \\
\text { generator }\end{array}$ & oscilloscope (-) & pulse & & Y-cut quartz (30 MHz) \\
\hline $\begin{array}{l}\text { Püttmer and Hauptmann } \\
\text { (1998), Püttmer et al. } \\
\text { (2000) }\end{array}$ & $\begin{array}{l}\text { analogue } \\
\text { signal } \\
\text { generator }\end{array}$ & $\begin{array}{l}\text { time-to-digital } \\
\text { converter (12 bit) }\end{array}$ & burst & 1 cycle & $\begin{array}{l}\text { lead metaniobate disk } \\
(2 \mathrm{MHz})\end{array}$ \\
\hline
\end{tabular}

The SNR dependency was discussed in Mak (1991), Higuti et al. (2001), Bjørndal and Frøysa (2008) and Bjørndal et al. (2008). Based on theoretical uncertainty considerations it was shown that the MRM is highly sensitive to noise. The more pulses included in the reflection coefficient calculation and the lower the SNR for each included pulse, the higher the uncertainty. Particularly in the case of the MRM, the SNR of $A_{\mathrm{e} 11}$ and $A_{\mathrm{e} 21}$ decreases dramatically when attenuation increases. Also, the SNR of $A_{\mathrm{e} 21}$ becomes quite low in the case of a low $r_{23}$. Additionally, in Mak (1991) the influence of diffraction correction uncertainties is discussed as a systematic error. Based on the fact that the RRM is independent of diffraction it was stated that the MRM is the least accurate method for calculating the reflection coefficient. Experimentally this general statement could not be proved so far; results of both MRM and RRM converged for the centre frequency of the transducer. Also the experimental results of Adamowski et al. (1995) showed similar errors for both methods. The comparison of MRM and TMSR in Bjørndal et al. (2008) showed a smaller systematic deviation from reference values for the MRM method. In Higuti et al. (2001) the statements are rested upon simulated signals with artificially added Gaussian white noise. In contrast to Bjørndal et al. (2008), who reported for a SNR of 50 an uncertainty of $25 \mathrm{~kg} \mathrm{~m}^{-3}$, in Higuti et al. (2001) for a similar SNR an error of only $1-5 \mathrm{~kg} \mathrm{~m}^{-3}$ was presented.

So far, Bjørndal (Bjørndal et al., 2008) is one of the few to have limited the sampling frequency and investigated the time resolution uncertainty by applying cubic spline approx- imation to synthetic $6 \mathrm{MHz}$ signals. Hence, the time resolution was increased from approximately $17 \mathrm{~ns}$ to $1 \mathrm{~ns}$ via mathematical approximation. In particular, the time domain results could be improved, and it can be assumed that the effect for signals of lower time resolution is even higher.

Unfortunately, none of the authors discussed the effect of systematic errors due to interference of subsequent pulses. Indeed, most authors state that clearly unaffected pulses are required for an accurate analysis, and cite several probe design considerations based upon a defined pulse length, but the truth is that the pulses are never diminished totally (see Püttmer et al., 1998, Figs. 7 and 8). As a basic rule, a pulse is regarded as terminated when the amplitude is below the noise level. But the subsequent signal is nothing more than a systematic oscillation hidden behind noise. Analysing those effects could help in separating such systematic errors from the signal.

The USV as a source of uncertainty often seems to be ignored. Most authors do not state how the speed of sound is determined and which accuracies could be reached (see Table 6). Generally the time of flight in the sample medium is determined and related to the propagation path. But often, particularly for small distances, the propagation path cannot be determined with adequate precision. The most chosen solutions to reach a higher precision are calibration measurements with standards (Marczak, 1997; Bjørndal et al., 2008; Higuti et al., 2001; Higuti and Adamowski, 2002b; Adamowski et al., 1998), which might become quite laborious if thermal expansion of the propagation path is 
considered. Alternatively a material of low thermal expansion such as ZERODUR ${ }^{\circledR}$ (Bjørndal et al., 2008; Hoppe et al., 2003) could be used. In a range of $\pm 25 \mathrm{~K}$ the thermal expansion can be neglected within an USV error of $0.2 \mathrm{~m} \mathrm{~s}^{-1}$. Standard for the time-of-flight determination is the cross correlation which can be applied in the time domain (Adamowski et al., 1995, 1998) or frequency domain (Deventer and Delsing, 1997). The great advantage of BRTs is the provision of a stable reference pulse that can be compared to echo pulses. Therefore the time-of-flight determination in pulse echo mode is independent of electronics time jitter. The only problematic parameter is the time resolution. When a simple cross correlation is applied, the time-of-flight resolution is still dependent on the sampling rate. For example, providing sampled data of $100 \mathrm{MHz}$ sampling rate leads to a $1 \mathrm{~m} \mathrm{~s}^{-1}$ velocity resolution for a $23 \mathrm{~mm}$ propagation path (Adamowski et al., 1995). That might be the reason why most researchers oversample the data. In fact, mathematical approximation is a feasible solution to achieve higher accuracies with less time resolution (Hoche et al., 2011; Hoppe et al., 2001). Apart from that, when echo detection in pulse echo mode becomes problematic (e.g. highly absorptive liquids, superposition of buffer reflections and echo pulses) often transmission measurements are necessary, which increases the uncertainties and the effort in technical equipment and analysis.

In fact, an accuracy of $0.1 \mathrm{~m} \mathrm{~s}^{-1}$ is reachable applying state-of-the-art technologies and methods, and the sound velocity is not actually the most critical source of uncertainty. Analysing the partial derivatives of Eq. (8) according to the propagation of uncertainties, one reaches the following: for $c_{1}$,

$\frac{\partial \rho_{2}}{\partial c_{1}} \Delta c_{1}=\frac{\rho_{1}\left(1+r_{\mathrm{A}}\right)}{c_{2}\left(1-r_{\mathrm{A}}\right)} \Delta c_{1}$

for $c_{2}$,

$\frac{\partial \rho_{2}}{\partial c_{2}} \Delta c_{2}=-\frac{\rho_{1} c_{1}\left(1+r_{\mathrm{A}}\right)}{c_{2}^{2}\left(1-r_{\mathrm{A}}\right)} \Delta c_{2}$

for $\rho_{1}$,

$\frac{\partial \rho_{2}}{\partial \rho_{1}} \Delta \rho_{1}=\frac{c_{1}\left(1+r_{\mathrm{A}}\right)}{c_{2}\left(1-r_{\mathrm{A}}\right)} \Delta \rho_{1}$

and for $r_{\mathrm{A}}$,

$\frac{\partial \rho_{2}}{\partial r_{\mathrm{A}}} \Delta r_{\mathrm{A}}=\frac{2 c_{1} \rho_{1}}{c_{2}\left(1-r_{\mathrm{A}}\right)^{2}} \Delta r_{\mathrm{A}}$.

The calculated proportions of uncertainties for different assumed errors are shown in Table 5. In the first row of uncertainties a constant error of $0.1 \%$ is assumed for all variables. The uncertainty examination shows that the contribution of reference values and measured sound velocity are comparable, while the contribution from the reflection coefficient is comparably small. Unfortunately the reachable reflection coefficient accuracies have not been reported so far. In the second row of uncertainties, realistic errors are assumed. The
Table 5. Contributed uncertainties of the relevant variables: buffer density, buffer sound velocity, sample medium sound velocity and reflection coefficient, with PMMA being the buffer and water being the sample medium.

\begin{tabular}{lrrrr}
\hline & $\rho_{1}\left[\mathrm{~kg} \mathrm{~m}^{-3}\right]$ & $c_{1}\left[\mathrm{~m} \mathrm{~s}^{-1}\right]$ & $c_{2}\left[\mathrm{~m} \mathrm{~s}^{-1}\right]$ & $r_{\mathrm{A}}$ \\
\hline value & 1181.77 & 2764.92 & 1482.38 & -0.3766 \\
error 1 & $\pm 0.1 \%$ & $\pm 0.1 \%$ & $\pm 0.1 \%$ & $\pm 0.1 \%$ \\
uncertainty 1 & \pm 0.998 & \pm 0.998 & \pm 0.998 & \pm 0.438 \\
{$\left[\mathrm{~kg} \mathrm{~m}^{-3}\right]$} & & & & \\
error 2 & $\pm 1 \mathrm{~kg} \mathrm{~m}^{-3}$ & $\pm 0.2 \mathrm{~m} \mathrm{~s}^{-1}$ & $\pm 0.2 \mathrm{~m} \mathrm{~s}^{-1}$ & $\pm 1 \mathrm{E}-04$ \\
uncertainty 2 & \pm 0.085 & \pm 0.007 & \pm 0.013 & \pm 0.116 \\
{$\left[\mathrm{~kg} \mathrm{~m}^{-3}\right]$} & & & & \\
\hline
\end{tabular}

reflection coefficient of error was estimated from theoretical considerations and uncertainties. The error contribution of sound velocity and density is still small, and the reported accuracies are sufficient to reach acceptable density uncertainties. But the contribution of a realistic reflection coefficient error to the density uncertainty is comparatively high, particularly considering that the reflection coefficient can result from several amplitude errors. For the coupled PMMAwater a density uncertainty of $0.25 \mathrm{~kg} \mathrm{~m}^{-3}$ can be expected overall. This uncertainty is still high compared to existing reference analytics such as the vibrating U-tube (see Table 6), but seems sufficient to use the BRTs as a monitoring tool in bioprocesses of small density change (see Table 1).

The most important uncertainty contribution which controls every influencing factor discussed so far is the temperature. The temperature accuracy affects the calibration measurements of the propagation path and buffer material's properties. Moreover, the temperature error affects uncertainties of temperature-dependent reference models as provided by the literature or certified reference standards. Using, for example, Marzcak's (Marczak, 1997) model to calculate the speed of sound of water at $20^{\circ} \mathrm{C}$, a $0.1 \mathrm{~K}$ temperature bias results in a $0.3 \mathrm{~m} \mathrm{~s}^{-1}$ USV bias, but only $0.03 \mathrm{~m} \mathrm{~s}^{-1}$ bias for a $0.01 \mathrm{~K}$ temperature bias. Due to the high impact of temperature on all relevant parameters, a temperature accuracy of at least $\pm 0.01 \mathrm{~K}$ is recommended. Most non-invasive temperature measurement techniques are too inaccurate and expensive (Childs et al., 2000). The standard for invasive temperature measurement is still the electrical resistance thermometry. In general, accuracies below $0.1 \mathrm{~K}$ can be achieved only through individual calibration regardless of the material. For highly accurate measurements, 4-wire systems, voltage reversal and low resistances are recommended.

The temperature also influences the dimensions and properties of the used materials, the characteristics of the sound field and even the properties of the PEM. So it is quite understandable that most authors have restricted their investigations to a constant temperature. In turn, the results of these works have to be evaluated with respect to the reported temperature stability. While in Bjørndal et al. (2008) a stability 
Table 6. Accuracies of involved measurement principles as published by different authors.

\begin{tabular}{|c|c|c|c|c|}
\hline Reference & $\begin{array}{l}\text { Density } \\
\text { reference }\end{array}$ & $\begin{array}{l}\text { Reference } \\
\text { accuracy } \\
\left(\mathrm{kg} \mathrm{m}^{-3}\right)\end{array}$ & $\begin{array}{l}\text { Measurement } \\
\text { points/temperature } \\
\text { accuracy }\end{array}$ & $\begin{array}{l}\text { USV accuracy } \\
\left(\mathrm{m} \mathrm{s}^{-1}\right)\end{array}$ \\
\hline $\begin{array}{l}\text { Adamowski et al. } \\
(1995,1998)\end{array}$ & pycnometer & $\pm 0.3 \mathrm{~kg} \mathrm{~m}^{-3}$ & $-/ \pm 0.5 \mathrm{~K}$ (varying) & 1.0 \\
\hline Bjørndal et al. (2008) & $\begin{array}{l}\text { literature/ } \\
\text { standards }\end{array}$ & $\pm 0.10 \mathrm{~kg} \mathrm{~m}^{-3}$ & $\begin{array}{l}-/ \pm 0.01 \mathrm{~K} \\
\text { (constant) }\end{array}$ & - \\
\hline $\begin{array}{l}\text { Deventer and Delsing } \\
\text { (1997) }\end{array}$ & literature & - & $\begin{array}{l}2 \text { points } / \pm 0.01 \mathrm{~K} \\
\text { (varying) }\end{array}$ & - \\
\hline $\begin{array}{l}\text { Greenwood et al. } \\
\text { (1999) }\end{array}$ & $\begin{array}{l}\text { volume } \\
\text { weighting }\end{array}$ & - & 3 points/- (varying) & - \\
\hline $\begin{array}{l}\text { Bamberger and Green- } \\
\text { wood }(2004 a, b)\end{array}$ & $\begin{array}{l}\text { volume } \\
\text { weighting }\end{array}$ & - & $-1-$ & - \\
\hline $\begin{array}{l}\text { McClements and Fairly } \\
(1991,1992)\end{array}$ & $\begin{array}{l}\text { vibrating } \\
\text { U-tube }\end{array}$ & $\pm 0.10 \mathrm{~kg} \mathrm{~m}^{-3}$ & $-/ 0.1$ (constant) & 0.5 \\
\hline $\begin{array}{l}\text { Papadakis et al. (1973); } \\
\text { Papadakis (1968) }\end{array}$ & - & - & $-1-$ & - \\
\hline $\begin{array}{l}\text { Püttmer and } \\
\text { Hauptmann (1998), } \\
\text { Püttmer et al. (2000) }\end{array}$ & $\begin{array}{l}\text { vibrating } \\
\text { U-tube }\end{array}$ & $\pm 0.10 \mathrm{~kg} \mathrm{~m}^{-3}$ & 1 point/- (constant) & - \\
\hline
\end{tabular}

of $\pm 0.04 \mathrm{~K}$ was reached, Adamowski et al. (1995) reported only $\pm 0.5 \mathrm{~K}$.

Additionally, temperature gradients have to be considered. Most researchers try to avoid gradients and control not only the temperature of the sample medium but also the environmental temperature (Bjørndal et al., 2008; Higuti et al., 2007). The procedure is acceptable for highly accurate validations but of low relevance for any practical application. In real process application often the sample medium or the environmental medium temperature varies, in the worst case even both. While the temperature of the sample medium is often controlled or behaves in a predictable way, the environmental temperature does not. Depending on the time of the year, the daytime, the local weather and the location and construction of the facility, the environmental temperature can vary in a range of \pm 5 to $\pm 20 \mathrm{~K}$. The point is that, in reality, there will be temperature gradients which are generally not constant, so the gradients have to be considered. Furthermore, the temperature control of the buffer is only a solution when the sample medium is also of constant temperature.

The methods that are affected most by temperature gradients are the ARM and RRM. When reference and calibration measurements are executed at different temperatures or gradients, the error can increase enormously. As stated before, temperature control is often not an acceptable solution and often not stable enough; therefore two options remain either the calibration for all relevant temperatures and gradients, which is extremely laborious, or an additional probe that determines parallel, under identical conditions to the reference values (Greenwood, 2000; Greenwood et al., 1999). Indeed, the parallel reference measurement minimizes the uncertainty caused by temperature gradients, but introduces new uncertainty sources due to the use of two excitation electronics, sender, receiver, and coupling systems that might be not identical. In the case of an MRM as proposed by Deventer and Delsing (2001b), temperature differences between sample medium and buffer rod interface temperature have to be considered. Therefore both should be monitored continuously. Similar effects have to be considered for propagation path calibrations (Higuti et al., 2007) and varying dynamic behaviour due to temperature changes of different magnitude which results in hysteresis effects (Deventer and Delsing, 2001a; Higuti et al., 2007).

In fact, there is another temperature gradient that has not been considered so far - the temperature gradient in the sample medium. As long as there is a temperature difference between sample medium and environment, there will be a gradient at the buffer-liquid interface, which implies three major issues:

1. The temperature variation over the sound propagation path influences the accuracy of the sound velocity measurement. In general, the properties vary with propagation path, and so does the sound velocity. In the end, the measured velocity, $\mathrm{USV}_{\mathrm{p}}$ represents the average of all variations. For a known temperature dependency of 
the velocity, $\mathrm{USV}(T)$ and a known temperature gradient $T(x)$ over the propagation path $x$, the relation can be described as follows:

$\mathrm{USV}_{\mathrm{p}}=\frac{1}{T\left(x_{2}\right)-T\left(x_{1}\right)} \int_{x 1}^{x 2}\left[U S V(T(x)) \cdot \frac{\partial T(x)}{\partial x}\right] \mathrm{d} x$.

The main conclusion of this expression is that if one wants to determine the temperature that fits to the measured USV, or vice versa, one has to determine the temperature at the right position or the mean temperature over the propagation path.

2. Equation (56) only introduces the general problem. The basic problem concerning the density determination is the combination of propagation path information and interfacial information. Knowing the temperature gradient means only that the measured sound velocity is not the sound velocity as it is next to the interface which is the relevant sound velocity for the reflection coefficient.

3. Thinking in terms of real process measurements, the temperature gradient cannot be considered to be simply a function of temperature difference. As soon as the sound velocity is measured in flows the gradient becomes dependent on the flow conditions.

To summarize, it can be expected that highly accurate measurements require multiple-point temperature measurements (see Table 6: Deventer and Delsing, 1997 and Greenwood et al., 1999) to gather all relevant temperatures and to estimate the gradients. Relevant temperature-dependent validations of ultrasound-based density determination are published in Adamowski et al. (1998), Greenwood and Bamberger (2002), Higuti et al. (2007), Deventer and Delsing (1997) and Deventer and Delsing (2001a).

The only method that can be assumed to be independent of gradients in the sample medium is the ARM. The density is determined via RRMs at two different angles (Eq. 41). The sound velocity can be calculated as an additional parameter from the determined density, but is not necessary for the density determination. If Eq. (42) is used, the calculated sound velocity can be assumed to be the interfacial sound velocity of the sample medium. On the other hand, the density uncertainties of the ARM can be assumed to be even more complex than presented in Eqs. (52)-(55). And, in case the sound velocity is not determined by the TOF-distance relation but by Eq. (42), the sound velocity uncertainty becomes similar in complexity.

The last point concerning the temperature-related uncertainties will be the temperature dependency of transducers and PEM. Most transducers possess a matching layer or wear plate. The transmission through such layers clearly is temperature dependent and can be described in terms of wavelength and layer thickness. Furthermore, for quartz crystals and piezocermic materials, it is known that the resonance behaviour changes with temperature (Hammond and Benjaminson, 1965; Yang, 2006). This effect can actually be used to measure the temperature. Once an MRM is used or the RRM and ARM are calibrated for different temperatures, those influences can be neglected in terms of attenuation or varying transmission coefficients, but the frequency behaviour might change significantly. Consequently, signal-processing methods in the frequency domain possibly have to be modified to consider temperature-dependent variations, particularly the single-frequency method (see Eq. 46).

\subsection{Relevant errors for industrial conditions}

This section discusses errors which are especially relevant for industrial applications. First of all, errors due to thin layers, which may represent coupling layers, matching layers or buffer surface deposits, will be discussed. Surface deposits might be applied as a protective layer or might appear as a result of fouling.

In Püttmer et al. (1999), the focus is on investigation of surface deposits by simulations via SPICE. After validation with polystyrene layers of varying thickness, the developed model was applied for materials of varying acoustic impedance and thickness. Scattering effects due to nonplane surfaces have been neglected. The results show that for layers of impedance lower than the buffer material and $\lambda / 100(\lambda / 50)$ thickness, the error of the sample medium's acoustic impedance can reach up to $0.5 \%$ (2.6\%); the USV error up to $0.05 \%(0.1 \%)$. For layers of impedance higher than the buffer material, the error increases rapidly. It is stated that deposits of low acoustic impedance such as polymers can be tolerated with a thickness up to $\lambda / 50$.

In Deventer (2003) also the influence of fouling deposits is investigated via a PSPICE model. Commensurate with a different probe design, the effects of deposits are simulated for a PMMA buffer instead of quartz glass (Püttmer et al., 1999). For the deposit material a density of $1500 \mathrm{~kg} \mathrm{~m}^{-3}$ and a sound velocity of $3000 \mathrm{~m} \mathrm{~s}^{-1}$ was assumed and thicknesses of $0.5,1$ and $2 \mu \mathrm{m}$ have been investigated. It was stated that, compared to a clean surface, the amplitude difference is quite high, but changing the layer thickness results only in small changes. While comparing the results with those of Püttmer et al. (1999), it was assumed that the model might be inconsistent. But comparing the details of both publications explains the difference: (1) in Püttmer et al. (1999) layer thicknesses relative to wavelength in the deposit material are investigated, which would correspond more likely to 8 and $17 \mu \mathrm{m}$ layer thicknesses in the case of Deventer (2003). (2) In Püttmer et al. (1999) no results of amplitude changes but errors in the determination of acoustic impedance and sound velocity are presented. (3) Checking the presented results of Püttmer et al. (1999) for impedances higher than the buffer materials, as investigated in Deventer (2003), one can assume that the amplitude difference is quite high compared to clean 
surface. Thus, based on the information given in Deventer (2003), no inconsistency is noticeable.

In Higuti et al. (2006) a model of acoustic or electroacoustic transmission lines was developed. The model was validated experimentally with signals from the true measurement cell, but without deposits. Metallization layers on the PVDFreceiver surface, varying thicknesses of the PVDF receiver, varying coupling layers and deposits on the buffer surface have been investigated. The thickness of the metallization layers was reported to be around $500 \AA$. In contrast to Deventer (2003) it was stated that layer thicknesses up to $1 \mu \mathrm{m}$ do not introduce significant changes in the signals, and their effects can be neglected. In the case of the receiver thickness, the pulse centre frequency changes with temperature, while the bandwidth remains constant. It is shown that layer thickness variations significantly change the frequency domain information, which might result in errors $>2 \%$ when applying the single-frequency approach. The error can be minimized by using the energy method and time delay compensation. The density error was kept within $\pm 0.2 \%$ for receiver thickness variations and within $\pm 0.1 \%$ for coupling layer variations up to $50 \mu \mathrm{m}$. Deposit results have been presented for varying thickness and different materials. For all presented materials the density error does not exceed $0.2 \%$ up to $2 \mu \mathrm{m}$ layer thickness. For higher thicknesses the error quickly reaches $6 \%$ and more.

Actually, neither in Püttmer et al. (1999) nor in Deventer (2003) or Higuti et al. (2006) is the relevance of the assumed fouling properties and layer thicknesses discussed. For milk fouling layers, for example, a layer thickness of 500-700 $\mu \mathrm{m}$ and an impedance of 2.97 MRayl has been reported (Wallhäußer et al., 2009). Hence, concerning the impedance of biological fouling layers, the assumption of lower acoustic impedance seems to be correct for most buffer materials. Whether relevant thicknesses have been investigated so far is questionable. Generally it can be stated that not much is known about the acoustic properties of real fouling layers and that electrical analogous systems can be applied to investigate the influence of thin layer deposits under ideal conditions (Deventer, 2003; Higuti et al., 2006; Püttmer et al., 1999) and to simulate design aspects of probes with a few limitations (Deventer, 2004). In Püttmer et al. (1999) it is shown that the error due to thin layers can be reduced as long as the degree of fouling can be detected. Reference calibrations with air are proposed, while in Deventer (2003) it is recommended to detect fouling at higher frequencies via broadband transducers. Also, in Higuti et al. (2006) it is stated that a periodic calibration with a reference medium might be necessary.

Besides surface deposits, short-term variations of process variables might have an influence on the method's accuracy. The influence of temperature variations and measurement accuracy has already been discussed above. Also, the influence of varying flow condition on temperature gradients has already been indicated, but not the direct signal diversion due to a flow perpendicular to the propagation path. Generally it is assumed that the diversion can be neglected as long as the sound velocity in the medium is considerably higher than the flow velocity. Assuming a moderate flow of $5 \mathrm{~m} \mathrm{~s}^{-1}$ typically results in a diversion angle of $0.2^{\circ}$. In consequence, each molecule is distracted approximately $0.003 \mathrm{~mm}$ per $\mathrm{mm}$ propagation path while the signal propagates through the sample medium. First of all, the diversion results in an offset diffraction, and furthermore the angular difference from normal incidence causes a difference of approximately $0.1 \%$ in the reflection coefficient. Greenwood et al. (1999) investigated flow velocities up to $2.5 \mathrm{~m} \mathrm{~s}^{-1}$ and found that the varying flow conditions did not significantly affect the average density bias. In Adamowski et al. $(1995,1998)$ varying flow velocities up to $10 \mathrm{~m} \mathrm{~s}^{-1}$ were investigated. It was found that the experimental results are not affected by the flow rate. Indeed, changes of reflection coefficient, sound velocity and density appeared, but relative to the temperature variation, the observed deviations have been within the precision range of the method. It is reported that cavitation occurred for mean flow velocities above $10 \mathrm{~m} \mathrm{~s}^{-1}$, and for this reason the results became inconsistent. Further issues might occur in the case of non-homogenous suspensions or bubbly flow. As correctly stated by Schäfer et al. (2006), the measurement effect bases on reflection at interfaces. Non-homogenous distributions of solid or gaseous objects across the interface would lead to a certain error. In Greenwood and Bamberger (2002) the feasibility of the ARM for homogenous suspensions was proven. The influence of bubbly flow was also investigated, and it was reported that three of the six investigated instruments have been significantly affected by the air feed. It can be assumed that generally the bubble dependency depends on the design and placement of the probe. As long as the bubbles do not adhere to the interface, no significant effect on the reflection coefficient should be noticeable. For the ARM also, the sound velocity determination only depends on the interfacial information. In the case of the other methods the situation for the sound velocity is quite different. Depending on the amount of air inside a certain volume, the density and compressibility change:

$$
\begin{aligned}
& \rho=\frac{\left(M_{1}+M_{2}\right)}{\left(V_{1}+V_{2}\right)}, \\
& \kappa=\frac{\left(\kappa_{1} V_{1}+\kappa_{2} V_{2}\right)}{\left(V_{1}+V_{2}\right)},
\end{aligned}
$$

where $M$ and $V$ represent the mass and volume and the indices indicate the particular phase. According to Eq. (1) the sound velocity changes as a result. In Hoppe et al. (2002) it was stated that the bubbles operate like a high-pass filter. It was shown in Hoppe et al. (2001) that the amplitude and the zero crossing times of detected pulses decrease, but the arrival time of the signal does not change. It was further stated that the influence of gas bubbles on the speed-of-sound accuracy can be minimized by adequate signal processing. 
Generally the attenuation due to bubbles is frequency dependent. The bubble size governs the resonance frequency of a bubble, and therefore the bubble size distribution with respect to the main frequency defines the degree of attenuation (Carstensen and Foldy, 1947; Silberman, 1957; Fox et al., 1995). According to Eq. (3), also the acoustic impedance could be affected for disadvantageous bubble distributions. Henning et al. noticed only a change of impedance for high bubble intensities (Hoppe et al., 2002).

\section{Conclusions}

In the last decades, several research groups have investigated varying methods based on BRTs. The reported methods can be classified into four main groups: MRM, TRM, RRM and ARM. Each method holds characteristic advantages and disadvantages. ARM and RRM are perfectly suited for highly sound absorbing liquids but require calibration measurements. The RRM is only suited for moderate sound absorbing liquids, but does not require calibrations. The TRM can be ranked somewhere in between, but as with the ARM, the method requires an additional receiver, which introduces additional sources of uncertainty. Although the RRM was proven theoretically to be more sensitive to SNRcaused inaccuracies than any other method, the experimental results did not confirm the theoretical evaluations. Basically all methods are sensitive to temperature gradients. While for MRM it is sufficient to determine the accurate temperature at the interface in order to determine the correct acoustic impedances, in the case of ARM and RRM it might be necessary to calibrate the probe for all relevant temperature gradients. An appropriate correction seems to be possible, but so far has not been proven to work accurately.

The main design limitations result from intentions to avoid pulse superposition. Pure pulses can be guaranteed by avoidance and suppression of radial mode vibrations and adequate dimensioning with respect to the given pulse duration and material properties. In some cases additional near-field constraints might have influenced the chosen dimension. Although angular reflections within the near field might disturb the sound field in a way that one should prevent the assumption of plane wave propagation, the ARM as well as the RRM can be assumed to be widely unaffected by those phenomena as long as all changes of the sound field are considered in the calibration. In the case of MRM and TRM, diffraction correction often is a major requirement for adequate errors. Alternatively to corrections, large-aperture receivers can be used in some applications to minimize the error.

The published results show minimum achievable density errors of $0.15 \%$ for constant temperature and $0.4 \%$ for varying temperatures, which is sufficient to identify liquids of significant different density. The question if the reported errors are sufficient for a suitable control of a specific process or not in the end depends on the density variation that can be expected. Sensitive biotechnological processes such as yeast fermentation generally show a density variation of $<60 \mathrm{~kg} \mathrm{~m}^{-3}$, which results in density accuracy requirements of at least $1 \mathrm{~kg} \mathrm{~m}^{-3}$ or $0.1 \%$. In the case of density-based models for concentration measurements of multicomponent mixtures, an even lower error might be necessary.

The uncertainty analysis shows that errors in the reflection coefficient contribute significantly to the overall density error but has been investigated least so far, whereas the contributions of realistic errors of the sound velocities and buffer material's density are comparably low. Indeed, most authors neither state the accuracies of the sound velocities nor the accuracy of the reflection coefficient measurement. Although the few presented USV errors are $\geq 0.5 \mathrm{~m} \mathrm{~s}^{-1}$, state-of-the-art technologies can provide accuracies $\leq 0.1 \mathrm{~m} \mathrm{~s}^{-1}$ even for low sampling frequencies. Moreover, the buffer material's density can be determined with acceptable accuracies keeping the uncertainties of the sample liquid's density within the required accuracy. Consequently, improvements in the reflection coefficient determination are the right choice to improve the density accuracy. Main improvements are reached by increasing the SNR and improving the amplitude determination. Most authors apply signal averaging, which reduces the Gaussian noise. But averaging of the whole signal is only a feasible method as long as the signal acquisition rate is much higher than changes of process parameters. In the case of fast varying sound velocity, signal averaging can cause systematic errors. We assume that it might be better not to average the whole signal but only the relevant pulses after being centred to a characteristic location. Errors due to systematic changes in the frequency domain can be minimized by applying the integration method to an adequate frequency band. The temperature measurement is identified as another main source of error. Often the temperature at a certain position is required to calculate the buffer material's properties from reference polynomials. In addition, temperature gradients may occur, particularly during dynamic process changes. Thus, for real-time process application and exact validation it is necessary to measure the temperature as accurately as possible $(\leq \pm 0.01 \mathrm{~K})$ and to observe temperature gradients as they may arise. Altogether it seems possible to reach an accuracy of $\leq 1 \mathrm{~kg} \mathrm{~m}^{-3}$ even for dynamic conditions. At present, the remaining uncertainty could be a result of both the assumed simplifications for the reflection coefficient at solid-liquid interfaces or the technological limitations - state of the art is a 12-bit resolution at $1 \mathrm{GHz}$ sampling rate; a higher vertical resolution of 14 bit or more often results in significantly lower sampling rates.

A sensor system for real-time process application will have to be suitable to fulfil all involved task reaching, from generation of the excitation signal and sound signal capturing over temperature measurement and up to signal processing. To date, most of the basics have been investigated, but still final statements about which technology or method suits best a certain case of application are not possible. It is not 
known if simple peak excitations are sufficient or if bursts of a certain frequency are the best choice. It is not clear exactly if signals of a specified frequency require a certain sampling frequency in order to reach the desired density accuracy or not. Similar can be stated for the different signal-processing methods. Applying spline interpolation in the time domain might reach comparable results such as integration in the frequency domain. The big question is which one requires less computational effort. From the technological point of view it is clear that a vertical resolution of 12 bit or better is required to reach accurate results. For statements about electronic effort, computation power and the required memory, first the basic aspects of signal generation and signal processing have to be discussed in more detail. Definitely not all methodical options to determine the reflection coefficient via BRT have been investigated so far, but the basic rules are clear: minimization or correction of temperature gradients, and maximization of SNR.

Edited by: M. Jose da Silva

Reviewed by: three anonymous referees

\section{References}

Adamowski, J. C., Buiochi, C., Simon, C., Silva, E. C. N., and Sigelmann, R. A.: Ultrasonic measurement of density of liquids, J. Acoust. Soc. Am., 97, 354-361, 1995.

Adamowski, J. C., Buiochi, C., and Sigelmann, R. A.: Ultrasonic Measurement of Density of Liquids Flowing in Tubes, IEEE Transactions on Ultrasonics, Ferroelectrics, and Frequency Control, 45, 48-56, 1998.

Asher, R. C.: Ultrasonics in chemical analysis, Ultrasonics, 25 1719, 1987.

Bamberger, J. A. and Greenwood, M. S.: Measuring fluid and slurry density and solids concentration non-invasively, Ultrasonics, 42, 563-567, 2004a.

Bamberger, J. A. and Greenwood, M. S.: Non-invasive characterization of fluid foodstuffs based on ultrasonic measurements, Food Res. Int., 37, 621-625, 2004b.

Bjørndal, E. and Frøysa, K. E.: Acoustic Methods for Obtaining the Pressure Reflection Coefficient from a Buffer Rod Based Measurement Cell, IEEE Trans UFFC, 55, 1781-1793, 2008.

Bjørndal, E., Frøysa, K. E., and Engeseth, S. A.: A Novel Approach to Acoustic Liquid Density Measurements Using a Buffer Rod Based Measuring Cell, IEEE Trans UFFC, 55, 1794-1808, 2008.

Bolotnikov, M. F., Neruchev, Y. A., Melikhov, Y. F., Verveyko, V. N., and Verveyko, M. V.: Temperature Dependence of the Speed of Sound, Densities, and Isentropic Compressibilities of Hexane + Hexadecane in the Range of (293.15 to 373.15) K, J. Chem. Eng. Data 50, 1095-1098, 2005.

Carlson, J. E., Deventer, J., and Micella, M.: Accurate temperature estimation in ultrasonic pulse-echo systems, World Congress on Ultrasonics, Paris, 2003a.

Carlson, J. E., Deventer, J., Scolan, A., and Carlander, C.: Frequency and Temperature Dependence of Acoustic Properties of Polymers Used in Pulse-Echo Systems, IEEE ULTRASONICS SYMPOSIUM, 8030032, 885-888, 2003 b.
Carstensen, E. L. and Foldy, L. L.: Propagation of Sound Through a Liquid Containing Bubbles, J. Acoust. Soc. Am., 19, 481-501, 1947.

Childs, P. R. N., Greenwood, J. R., and Long, C. A.: Review of temperature measurement, Rev. Sci. Instrum., 71, 2959-2978, doi:10.1063/1.1305516, 2000.

Daridon, J. L., Lagourette, B., Xan, B., and Montel, F.: Petroleum characterization from ultrasonic measurement, J. Petrol. Sci. Eng., 19 281-293, 1998a.

Daridon, J. L., Lagrabette, A., and Lagourette, B.: Speed of sound, density, and compressibilities of heavy synthetic cuts from ultrasonic measurements under pressure, J. Chem. Thermodynam., 30, 607-623, 1998b.

Davis, L. A. and Gordon, R. B.: Compression of Mercury at High Pressure, J. Chem. Phys., 46, 2650-2660, 1967.

Deventer, J.: Detection of, and compensation for error inducing thin layer deposits on an ultrasonic densitometer for liquids, Instrumentation and Measurement Technology Conference 2003, 648651, 2003.

Deventer, J.: One dimensional modeling of a step-down ultrasonic densitometer for liquids, Ultrasonics, 42, 309-314, 2004.

Deventer, J. and Delsing, J.: An Ultrasonic Density Probe, IEEE ULTRASONICS SYMPOSIUM, 1997.

Deventer, J. and Delsing, J.: Thermostatic and Dynamic Performance of an Ultrasonic Density Probe, IEEE Trans UFFC, 48, 675-682, 2001a.

Deventer, J. and Delsing, J.: Thermostatic and Dynamic Performance of an Ultrasonic Density Probe, IEEE Trans. UFFC, 48, 675-682, 2001b.

Esperança, J. M. S. S., Visak, Z. P., Plechkova, N. V., Seddon, K. R., Guedes, H. J. R., and Rebelo, L. P. N.: Density, Speed of Sound, and Derived Thermodynamic Properties of Ionic Liquids over an Extended Pressure Range. 4. [C3mim][NTf2] and [C5mim][NTf2], J. Chem. Eng. Data, 51, 2009-2015, 2006.

Fisher, B., Magpori, V., and von Jena, A.: Ultraschall (US)Dichtemesser mm Messen der spezifischen Dichte eines Fluid, EP 0483491 81, Europe, 1995.

Fox, F. E., Curley, S. R., and Larson, G. S.: Phase Velocity and Absorption Measurements in Water Containing Air Bubbles, The J. Acoust. Soc. Am., 27, 534-539, 1995.

Greenwood, M. S.: Ultrasonic fluid densitometer having liquid/wedge and gas/wedge interfaces, 6, 082, 181, United States, 2000.

Greenwood, M. S. and Bamberger, J. A.: Ultrasonic sensor to measure the density of a liquid or slurry during pipeline transport, Ultrasonics, 40, 413-417, 2002.

Greenwood, M. S. and Bamberger, J. A.: Self-Calibrating Sensor for Measuring Density Through Stainless Steel Pipeline Wall, J. Fluid. Eng., 126, 189-192, 2004.

Greenwood, M. S., Skorpik, J. R., Bamberger, J. A., and Harris, R. V.: On-line Ultrasonic Density Sensor for Process Control of Liquids and Slurries, Ultrasonics, 37, 159-171, 1999.

Greenwood, M. S., Adamson, J. D., and Bamberger, J. A.: Longpath measurements of ultrasonic attenuation and velocity for very dilute slurries and liquids and detection of contaminates, Ultrasonics, 44, e461-e466, 2006.

Guilbert, A. R. and Sanderson, M. L.: A novel ultrasonic mass flowmeter for liquids, IEE colloquium on: Advances in Sensors for Fluid Flow Measurement, London, 1996. 
Hale, J. M.: Ultrasonic density measurement for process control, Ultrasonics, 26, 356-357, 1988.

Hammond, L. D. and Benjaminson, A.: The Linear Quartz Thermometer - a New Tool for Measuring Absolute and Difference Temperatures, Hewlett-Packard Journal, 16, 1965.

Henning, B., Prange, S., Dierks, K., Daur, C., and Hauptmann, P.: In-line concentration measurement in complex liquids using ultrasonic sensors, Ultrasonics, 38, 799-803, 2000.

Higuti, R. T. and Adamowski, J. C.: Ultrasonic Densitometer Using a Multiple Reflection Technique, IEEE Trans UFFC, 49, 12601268, 2002a.

Higuti, R. T. and Adamowski, J. C.: Ultrasonic densitometer using a multiple reflection technique, IEEE Trans. Ultrason., Ferroelect., Freq. Contr., 49, 1260-1268, 2002b.

Higuti, R. T., Montero de Espinosa, F. R., and Adamowski, J. C.: Energy method to calculate the density of liquids using ultrasonic reflection techniques, Proc. IEEE Ultrason. Symp., 319322, 2001.

Higuti, R. T., Buiochi, C., Adamowski, J. C., and Espinosa, F. M.: Ultrasonic density measurement cell design and simulation of non-ideal effects, Ultrasonics, 44, 302-309, 2006.

Higuti, R. T., Galindo, B. S., Kitano, C., Buiochi, C., and Adamowski, J. C.: Thermal Characterization of an Ultrasonic Density-Measurement Cell, IEEE Transactions on Instrumentation and Measurement, 56, 924-930, 2007.

Hoche, S., Hussein, W. B., Hussein, M. A., and Becker, T.: Time-of$\mathrm{f}$ light prediction for fermentation process monitoring, Eng. Life Sci., 11, 1-12, 2011.

Hoppe, N., Schönfelder, G., Püttmer, A., and Hauptmann, P.: Ultrasonic density sensor - Higher accuracy by minimizing error influences, Proc. IEEE Ultrason. Symp., 361-364, 2001.

Hoppe, N., Schönfelder, G., and Hauptmann, P.: UltraschallDichtesensor für Flüssigkeiten - Eigenschaften und Grenzen, Technisches Messen, 3, 131-137, 2002.

Hoppe, N., Püttmer, A., and Hauptmann, P.: Optimization of Buffer Rod Geometry for Ultrasonic Sensors with Reference Path, IEEE Trans UFFC, 50, 170-178, 2003.

Jensen, B. R.: Measuring equipment for acoustic determination of the specific gravity of liquids, 4, 297, 608, United States, 1981.

Kaatze, U., Eggers, F., and Lautscham, K.: Ultrasonic velocity measurements in liquids with high resolution - techniques, selected applications and perspectives, Meas. Sci. Technol., 19, 1-21, doi:10.1088/0957-0233/19/6/062001, 2008.

Kaduchak, G. and Sinha, D. N.: Apparatus and method for remote, noninvasive characterization of structures and fluids inside containers, 8, 186, 004 B1, United States, 2001.

Kell, G. S.: Density, Thermal Expansivity, and Compressibility of Liquid Water from $0^{\circ}$ to $150^{\circ} \mathrm{C}$ : Correlations and Tables for Atmospheric Pressure and Saturation Reviewed and Expressed on 1968 Temperature Scale, J. Chem. Eng. Data, 20, 97-105, 1975.

Kim, J. O. and Bau, H. H.: Instrument for simultaneous measurement of density and viscosity, Rev. Sci. Instrum., 60, 1111-1115, 1989.

Krautkramer, J. and Krautkramer, H.: Ultrasonic Testing of Materials, 3rd edn. ed., Springer-Verlag, New York, 1983.

Kulmyrzaev, A., Cancelliere, C., and McClements, D. J.: Characterization of aerated foods using ultrasonic reflectance spectroscopy, J. Food Eng., 46, 235-241, 2000.
Kuo, H. L.: Variation of Ultrasonic Velocity and Absorption with Temperature and Frequency in High Viscosity Vegetable Oils, Japanese Journal of Applied Physics, 10, 167-170, 1971.

Kushibiki, J., Akashi, N., Sannomiya, T., Chubachi, N., and Dunn, F.: VHF/UHF range bioultrasonic spectroscopy system and method, IEEE Trans. Ultrason., Ferroelec. Freq. Contr., 42, 1028-1039, 1995.

Kushibiki, J., Okabe, R., and Arakawa, M.: Precise measurements of bulk-wave ultrasonic velocity dispersion and attenuation in solid materials in the VHF range, J. Acoust. Soc. Am., 113, 3171-3178, 2003.

Lach, M., Platte, M., and Ries, A.: Piezoelectric materials for ultrasonic probes, NDTnet, 1, 1996.

Lynnworth, L. C. and Pedersen, N. E.: Ultrasonic mass flowmeter, Proc. IEEE Ultrason. Symp., 87-90, 1972.

Lynnworth, L. C.: Ultrasonic nonresonant sensors, in: Sensors A Comprehensive Survey, edited by: Göpel, W., Hesse, J., and Zemel, J. N., Mechanical Sensors, VCH Publishers Inc., New York, 311-312, 1994.

Mak, D. K.: Comparison of various methods for the measurment of reflection coefficient and ultrasonic attenuation, British Journal of NDT, 33, 441-449, 1991.

Marczak, W.: Water as standard in the measurements of speed of sound in liquids, J. Acoust. Soc. Am., 102, 2776-2779, 1997.

Marks, G. W.: Acoustic Velocity with Relation to Chemical Constitution in Alcohols, The Journal of the Acoustical Society of America, 41, 103-117, 1976.

Mason, P., Baker, W. O., McSkimin, H. J., and Bepiss, J. H.: Measurement of Shear Elasticity and Viscosity of Liquids at Ultrasonic Frequencies, Phys. Rev., 75, 936-946, 1949.

Matson, J., Mariano, C. F., Khrakovsky, O., and Lynnworth, L. C.: Ultrasonic Mass Flowmeters Using Clamp-On or Wetted Transducers, 5th International Symposium on Fluid Flow Measurement, Arlington, Virginia, 2002,

McClements, D. J.: Ultrasonic Characterization of Foods and Drinks: Principles, Methods, and Applications, Critical Reviews in Food Science and Nutrition, 37, 1-46, 1997.

McClements, D. J. and Fairly, P.: Ultrasonic pulse echo reflectometer, Ultrasonics 29, 58-62, 1991.

McClements, D. J. and Fairly, P.: Frequency scanning ultrasonic pulse echo reflectometer, Ultrasonics, 30, 403-405, 1992.

Mc Gregor, K. W.: Methods of Ultrasonic Density Measurement, Australasian Instrumentation and Measurement Conference, Adelaide, S. Aust., 1989.

Moore, R. S. and McSkimin, H. J.: Physical Acoustics, Academic Press, New York, 167-242, 1970.

O'Neil, H. T.: Reflection and Refraction of Plane Shear Waves in Viscoelastic Media, Phys. Rev., 75, 928-935, 1949.

Papadakis, E. P.: Correction for Diffraction Losses in the Ultasonic Field of a Piston Source, J. Acoust. Soc. Am., 31, 150-152, 1959.

Papadakis, E. P.: Buffer-Rod System for Ultrasonic Attenuation Measurements, J. Acoust. Soc. Am., 44, 1437-1441, 1968.

Papadakis, E. P., Fowler, K. A., and Lynnworth, L. C.: Ultrasonic attenuation by spectrum analysis of pulses in buffer rods: Method and diffraction corrections, J. Acoust. Soc. Am., 53, 1336-1343, 1973.

Pope, N. G., Veirs, D. K., and Claytor, T. N.: Fluid Density and Concentration Measurment using noninvasive in situ ultrasound 
resonance interferometry, Ultrasonics Symposium, 1992, 855858, 1992.

Pope, N. G., Veirs, D. K., and Claytor, T. N.: Fluid density and concentration measurment using noninvasive in situ ultrasonic resonance interferometry, 5, 359, 541, United States, 1994.

Povey, M. J. W. and McClements, D. J.: Ultrasonics in Food Engineering. Part I: Introduction and Experimental Methods, J. Food Eng., 8, 217-245, 1988.

Püttmer, A. and Hauptmann, P.: Ultrasonic density sensor for liquids, Proc. IEEE Ultrason. Symp., 497-500, 1998.

Püttmer, A., Lucklum, R., Henning, B., and Hauptmann, P.: Improved ultrasonic density sensor with reduced diffraction influence, Sensors Actuators A, 67, 8-12, 1998.

Püttmer, A., Hoppe, N., Henning, B., and Hauptmann, P.: Ultrasonic density sensor-analysis of errors due to thin layers of deposits on the sensor surface, Sensor. Actuator., 76, 122-126, 1999.

Püttmer, A., Hauptmann, P., and Henning, B.: Ultrasonic density sensor for liquids, IEEE Trans. Ultrason., Ferroelec. Freq. Contr., 47, 85-92, 2000.

Raum, K., Ozguler, A., Morris, S. A., and O'Brien, W. D. J.: Channel Defect Detection in Food Packages Using Integrated Backscatter ultrasound Imaging, IEEE Trans UFFC, 45, 30-40, 1998.

Rychagov, M. N., Tereshchenko, S., Masloboev, Y., Simon, M., and Lynnworth, L. C.: Mass Flowmeters for Fluids with Density Gradient, IEEE Ultrasonics Symposium, 465-470, 2002.

Sachse, W.: Density determination of a fluid inclusion in an elastic solid from ultrasonic spectroscopy measurements, Proc. IEEE Ultrason. Symp., 716-719, 1974.

Saggin, R. and Coupland, J. N.: Concentration Measurement by Acoustic Reflectance, J. Food Sci., 66, 681-685, 2001.

Schäfer, R., Carlson, J. E., and Hauptmann, P.: Ultrasonic concentration measurement of aqueous solutions using PLS regression, Ultrasonics, 44, e947-e950, 2006.

Silberman, E.: Sound Velocity and Attenuation in Bubbly Mixtures Measured in Standing Wave Tubes, J. Acoust. Soc. Am., 29, 925-933, 1957.

Sinha, D. N.: Noninvasive identification of fluids by swept frequency acoustic interferometry, 5, 767, 407, United States, 1998.
Sinha, D. N. and Kaduchak, G.: Chapter 8: Noninvasive determination of sound speed and attenuation in liquids, in: Experimental Methods in the Physical Sciences, Academic Press, 307-333, 2001.

Swoboda, C. A., Frederickson, D. R., Gabelnick, S. D., Cannon, P. H., Hornestra, F., Yao, N. P., Phan, K. A., and Singleterry, M. K.: Development of an Ultrasonic Technique to Measure Specific Gravity in Lead-Acid Battery Electrolyte, IEEE Transactions on Sonics and Ultrasonics, 30, 69-77, 1983.

Van Sint Jan, M., Guarini, M., Guesalaga, A., Ricardo Perez-Correa, J., and Vargas, Y.: Ultrasound based measurements of sugar and ethanol concentrations in hydroalcoholic solutions, Food Control, 19, 31-35, 2008.

Vray, D., Berchoux, D., Delachartre, P., and Gimenez, G.: Speed of Sound in Sulfuric Acid Solution: Application to Density Measurement, Ultrasonics Symposium, 465-470, 1992.

Wallhäußer, E., Hussein, M. A., Hinrichs, J., and Becker, T.: The acoustic impedance - an indicator for concentration in alcoholic fermentation and cleaning progress of fouled tube heat exchangers, 5th International Technical Symposium on Food Processing, Monitoring Technology in Bioprocesses and Food Quality Management, Potsdam, Germany, 1 September, 2009.

Wang, H., Cao, Y., Zhang, Y., and Chen, Z.: The design of The ultrasonic liquid density measuring instrument, Third International Conference on Measuring Technology and Mechatronics Automation, 2011.

Wang, Z. and Nur, A.: Ultrasonic velocities in pure hydrocarbons and mixtures, J. Acoust. Soc. Am., 89, 2725-2730, 1991.

Williams, A. O. J. and Labaw, L. W.: Acoustic Intensity Distribution from a "Piston" Source, J. Acoust. Soc. Am., 16, 231-236, 1945.

Williams, A. O. J.: The Piston Source at High Frequencies, J. Acoust. Soc. Am., 23, 1-6, 1951.

Yang, J.: Chapter 10: Temperature Sensors, in: Analysis of Piezoelectric Devices, World Scientific Publishing Co. Pte. Ltd., Singapore, 371-386, 2006.

Żak, A., Dzida, M., Zorbȩski, M., and Ernst, S.: A high pressure device for measurements of the speed of sound in liquids, Rev. Sci. Instrum., 71, 1756-1765, 2000. 